\title{
Constructive uniformities of pseudometrics and Bishop topologies $^{1}$
}

\author{
IOSIF PETRAKIS
}

\begin{abstract}
We develop the first steps of a constructive theory of uniformities given by pseudometrics and study its relation to the constructive theory of Bishop topologies. Both these concepts are constructive, function-theoretic alternatives to the notion of a topology of open sets. After motivating the constructive study of uniformities of pseudometrics we present their basic theory and we prove a Stone-Čech theorem for them. We introduce the $f$-uniform spaces and we prove a Tychonoff embedding theorem for them. We study the uniformity of pseudometrics generated by some Bishop topology and the pseudo-compact Bishop topology generated by some uniformity of pseudometrics. Defining the large uniformity on reals we prove a "large" version of the Tychonoff embedding theorem for $f$-uniform spaces and we show that the notion of morphism between uniform spaces captures Bishop continuity. We work within BISH* , Bishop's informal system of constructive mathematics BISH extended with inductive definitions with rules of countably many premisses.
\end{abstract}

2010 Mathematics Subject Classification 03F60 (primary); 03F65 (secondary)

Keywords: uniform spaces, pseudometrics, Bishop spaces, constructive topology

\section{Uniformities of pseudometrics in constructive topology}

A uniformity of pseudometrics was the first notion of uniformity, which was introduced by Weil in [47] as a natural generalization of the notion of a metric. Shortly after, Tukey's uniformity of coverings and Bourbaki's unifomities of entourages were introduced in [45] and [8], respectively. Classically, these notions of uniform space are equivalent. As it is mentioned in Howes [22, page 43], "Weil's original approach was rather unwieldy and was soon replaced by (the) two others". Despite this prevailing view, today uniformities in the language of pseudometrics are still studied classically (see, for example, Pachl [29]). Moreover, as this is shown in the classic book [21], the notion of

\footnotetext{
${ }^{1}$ This paper is included in the Proceedings of the Fifth Workshop on Formal Topology, Institut Mittag-Leffler, June 2015 (editors Thierry Coquand, Maria Emilia Maietti and Erik Palmgren).
} 
uniformity which suits better to the classical theory of $C(X)$ is that of Weil's. In [21, page 216] Gillman and Jerison remark the following:

From our point of view, the most efficient approach to uniform spaces is by way of pseudometrics, as they provide us with a large supply of continuous functions Accordingly, we define a uniform structure to be a family of pseudometrics (satisfying appropriate closure conditions). This enables us to give complete proofs relatively quickly of all the facts about uniform spaces that are needed here.

Uniformities given by entourages have been studied extensively within the constructive theory of apartness spaces, developed mainly by Bridges and Vîţă in [13] (see also Bridges [14] for more recent results). ${ }^{2}$ This notion of uniformity is a set-theoretic one, which fits to the set-theoretic character of the notion of an apartness space. As far as we know, uniformities of coverings have not been studied constructively yet.

The constructive study of uniformities given by pseudometrics has a more complex history. ${ }^{3}$ Bishop defined a uniform space through pseudometrics ${ }^{4}$ in [4, pages 110-1], and this definition was repeated in [7, pages 124-5]. Although some fundamental properties of uniform spaces were given in [4] in the form of exercises, Bishop expressed a negative view towards the development of a constructive theory of uniformities given by pseudometrics. In [4, pages 349-51], Bishop makes the following comment.

A uniform space at first sight appears to be a natural and fruitful concept of a topological space. In fact, this is not the case. For instance, just to construct a compact uniform space $X$, such that the assumption that $X$ is metrizable leads to a contradiction, seems to be a hard problem. ... Of course, important constructively defined uniform spaces that are not necessarily metrizable exist: every locally convex space has a natural uniform structure. At first glance, the concept of a locally convex space would appear to be important for constructive mathematics, since examples exist in profusion.

\footnotetext{
${ }^{2}$ Richman has also studied constructively such uniformities in his unpublished work [41].

${ }^{3} \mathrm{We}$ confine our account to the study of uniformities of pseudometrics within Bishop-style constructive mathematics. For the study of uniformities of pseudometrics in formal topology we refer to Curi [17], Fox [19] and Kawai [24].

${ }^{4}$ Bishop's definition is more general than the one we use here (see Definition 2.4), although the property $\left(D_{2}\right)$ added here and also found in the classical literature (see for example [21, page 217]) is incorporated in Bishop's definition of a morphism between uniform spaces, and it corresponds to the closure of a Bishop topology of functions under uniform limits (see clause $\left(\mathrm{BS}_{4}\right)$ of Definition 4.2).
} 
However, in most cases of interest it seems to be unnecessary to make use of any deep facts from the general theory of convex spaces.

To Bishop's latter argument Bridges and Vîţă respond in [12, page 127], saying that ... the development of constructive analysis (in particular, aspects of the theory of operators) in recent years has greatly benefited from such a general theory... ${ }^{5}$

To justify his former argument, Bishop explains in [4, page 350] why the most obvious expected example of a non-metrizable compact uniform space, ie the product uniform space $X=[0,1]^{S}$ where $S$ is an uncountable set, cannot be shown to be compact uniform space, since it cannot be shown to be totally bounded uniform space, ie totally bounded with respect to all additions of the pseudometrics in the uniform structure of $X$. In our view though, this problem does not necessarily imply that the concept of a uniformity of pseudometrics is unnatural or unfruitful. Rather it forces one to find a notion of compact uniformity of pseudometrics that does not copy the definition of a compact metric and at the same time is reduced to it when the uniform space is a metric one. Such an enterprise with respect to compactness has been shown fruitful in formal topology (see Palmgren [30]), and in the theory of Bishop spaces (see Petrakis [36]). As we show in [33], [38] and [39], the constructive theory of metric spaces has also benefited from the general theory of Bishop spaces. For example, the fact that a non-zero bounded multiplicative linear functional on $C(K)$, where $K$ is a compact metric space, is determined by some point of $K$ (Proposition 8.25 in [7, page 382]) is proved in [7] within the theory of normed spaces, while in [39] it is a corollary within the theory of Bishop spaces. Note also that in Bishop's attempt to reconstruct some portion of general topology constructively, found in his unpublished manuscript [6], uniform spaces of pseudometrics play an important role, as ecclesiastical spaces - the main objects under study - are such uniform spaces equipped with a hierarchy, an appropriate collection of subsets of the main set.

In [13, page 178] it is commented that classical results such as (for example) that a uniformity of entourages is induced by a family of pseudometrics, or that a uniformity with a countable base of entourages is induced by a single pseudometric (see Bourbaki [9, Chapter IX] for a classical proof of these facts), are not expected to hold constructively. This cannot be seen though as an argument against the constructive study of uniformities of pseudometrics, since this is very often the case with constructive studies of concepts which have already been treated classically. In Berger, Ishihara, Palmgren and

\footnotetext{
${ }^{5}$ They mean a general theory of locally convex spaces, presented in Section 5.4 of [12]. See also the thesis [44] of Spitters for contributions in this theory.
} 
Schuster [3, page 975] it is noted that the hypothesis that the discrete uniformity $L(X)=\{U \subseteq X \times X \mid \Delta \subseteq U\}$, where $\Delta(X)=\{(x, x) \in X \times X \mid x \in X\}$, is induced by a set of pseudometrics $D$ on $X$, ie for every $U \in L$ there exist $d_{1}, \ldots d_{n} \in D$ and $\epsilon>0$ such that

$$
\left\{(x, y) \in X \times X \mid \forall_{1 \leq j \leq n}\left(d_{j}(x, y) \leq \epsilon\right)\right\} \subseteq U,
$$

implies the weak limited principle of omniscience in the form $\forall_{a, b \in \mathbb{R}}(a=b \vee \neg(a=b))$. Again, this fact cannot be considered as an argument against the development of the constructive theory of uniformities of pseudometrics, since the aim of such a theory is not to capture all classical results governing the relation between uniformities of entourages and uniformities of pseudometrics, a relation which is based on the fact that classically set-theoretic and function-theoretic objects are treated similarly. In constructive mathematics, though, function-theoretic objects behave better than set-theoretic ones.

What we want to emphasize here is that as the constructive study of uniformities of entourages fits to the constructive study of apartness spaces, the constructive study of uniformities of pseudometrics fits to the constructive study of Bishop spaces. As we try to show in the rest of this paper, uniformities of pseudometrics and Bishop topologies share the following characteristics.

(1) Both notions are function-theoretic.

(2) Their definitions have similar structure and induce similar function-theoretic notions of morphisms.

(3) They posses an intrinsic inductive character, which is represented in the concepts of the least uniformity generated by a given set of pseudometrics and of the least Bishop topology generated by a given set of real-valued functions.

(4) Their theories can be developed in parallel and within the same system BISH*, Bishop's informal system of constructive mathematics BISH (see Bishop [4], Bishop and Bridges [7], Beeson [2], Bridges and Richman [10] and Bridges and Vîţă [12]) extended with inductive definitions with rules of countably many premisses.

In [28] Myhill proposed the formal theory CST of sets and functions to codify BISH. He also took Bishop's inductive definitions in [4] (of Borel set and of function space, here called Bishop space) at face value and showed that the existence and disjunction properties of CST persist in the extended with inductive definitions system CST*, which can be considered as a formalization of $\mathrm{BISH}^{*}$. As another formalization of $\mathrm{BISH}^{*}$ one can consider the system CZF + REA + DC, where Aczel's regular extension axiom 
REA accommodates inductive definitions in CZF (see Lubarsky and Rathjen [26]) and DC denotes the axiom of dependent choice (see [10, page 12]). Here we describe the computational meaning of the theory of uniformities of pseudometrics (and of Bishop topologies) within the informal system BISH* .

\section{Basic notions and facts}

We present some first definitions and results necessary to the rest of the paper.

Definition 2.1 A setoid is a pair $\left(X,=_{X}\right)$, where $X$ is a set and $=_{X}$ is an equivalence relation on $X$. It is called inhabited if there is $x_{0} \in X$. If $\left(X,=_{X}\right),\left(Y,=_{Y}\right)$ are setoids, ${ }^{6}$ a function $f$ from $X$ to $Y$ is an operation such that $x={ }_{X} y \rightarrow f(x)={ }_{Y} f(y)$, for every $x, y \in X$ (see [7, page 15]). We denote by $\mathbb{F}(X, Y)$ the set of functions from $X$ to $Y$, which is equipped with the pointwise equality; by $\mathbb{F}(X)$ the set of all functions from $X$ to $\mathbb{R}$, where $\mathbb{R}$ is equipped with the standard equality (see [7, page 18]); and by $\mathbb{F}^{*}(X)$ the set of bounded elements of $\mathbb{F}(X)$. If $f, g \in \mathbb{F}(X)$ we define $f \leq g:=\forall_{x \in X}(f(x) \leq g(x))$. If $a \in \mathbb{R}$ we denote by $\bar{a}_{X}$ the constant function on $X$ with value $a$, and their set by $\operatorname{Const}(X)$.

Within the theory of uniform spaces of pseudometrics the main objects of study are the pseudometrics on $X$, while within the theory of Bishop spaces the main objects of study are the functions of type $X \rightarrow \mathbb{R}$. For the rest of this paper $X, Y$ denote inhabited setoids.

Definition 2.2 A pseudometric on $X$ is a mapping $d: X \times X \rightarrow[0,+\infty)$ such that $x=y \rightarrow d(x, y)=0, d(x, y)=d(y, x)$, and $d(x, y) \leq d(x, z)+d(z, y)$, for every $x, y, z \in X$. We denote by $\mathbb{D}(X)$ the set of all pseudometrics on $X$. If $d$ is a pseudometric on $X$, the pair $(X, d)$ is called a pseudometric space. A pseudometric $d$ on $X$ is called bounded if there exists some $M>0$ such that $d \leq \bar{M}_{X \times X}$. We denote by $\mathbb{D}^{*}(X)$ the set of bounded pseudometrics on $X$. If $f$ is a function of type $X \rightarrow \mathbb{R}$, the pseudometric $d_{f}$ induced by $f$ is defined by

$$
d_{f}(x, y):=d_{\mathbb{R}}(f(x), f(y))=|f(x)-f(y)|
$$

for every $x, y \in X$. The constant function $\overline{0}_{X \times X}$ on $X \times X$ is also a pseudometric, which we call the zero pseudometric on $X$. A pseudometric $d$ on $X$ is called non-zero if there exist $x_{0}, y_{0} \in X$ such that $d\left(x_{0}, y_{0}\right)>0$. If $d \in \mathbb{D}(X)$ and $x_{0} \in X$, the pseudodistance

\footnotetext{
${ }^{6}$ Usually we use for simplicity a single equality symbol for two setoids avoiding subscripts.
} 
at $x_{0}$ with respect to $d$ is the mapping $d_{x_{0}}: X \rightarrow[0, \infty)$, defined by $x \mapsto d\left(x, x_{0}\right)$ for every $x \in X$. A pseudometric $d$ is called a metric if $d(x, y)=0 \rightarrow x=y$ for every $x, y \in X$, and then the structure $(X, d)$ is called a metric space.

One could write the first definitional clause of a pseudometric as $\forall_{x \in X}(d(x, x)=0)$, avoiding in this way mentioning some equality on $X$. Since this is required though in the definition of a metric and of a separating set of pseudometrics (see Definition 3.1), we include the setoid structure of $X$ in Definition 2.2. If $d_{1}, d_{2}$ are two pseudometrics on $X$, it is immediate to see that $d_{1}+d_{2}$ and $d_{1} \vee d_{2}$ are pseudometrics on $X$, where $\left(d_{1} \vee d_{2}\right)(x, y)=d_{1}(x, y) \vee d_{2}(x, y)$ for every $x, y \in X$, and $a \vee b=\max \{a, b\}$ for every $a, b \in \mathbb{R}$. Addition and multiplication of real-valued functions are defined pointwisely.

Definition 2.3 If $d, e \in \mathbb{D}(X), \Delta \subseteq \mathbb{D}(X)$, and $\delta, \epsilon>0$, we define:

$$
\begin{aligned}
U(d, \delta, e, \epsilon) & :=\forall_{x, y \in X}(d(x, y) \leq \delta \rightarrow e(x, y) \leq \epsilon) \\
U(\Delta, e) & :=\forall_{\epsilon>0} \exists_{\delta>0} \exists_{d \in \Delta}(U(d, \delta, e, \epsilon)) \\
\bar{\Delta} & :=\{e \in \mathbb{D}(X) \mid U(\Delta, e)\}
\end{aligned}
$$

We call $\bar{\Delta}$ the pseudometric closure of $\Delta$, while if $\bar{\Delta}=\Delta$ we say that $\Delta$ is pseudometrically closed.

If $(X, \rho)$ is a metric space, $f: X \rightarrow \mathbb{R}$ is uniformly continuous with modulus of continuity $\omega_{f}$, ie

$$
\forall_{x, y \in X}\left(\rho(x, y) \leq \omega_{f}(\epsilon) \rightarrow|f(x)-f(y)| \leq \epsilon\right)
$$

and $g$ is just a function of type $X \rightarrow \mathbb{R}$, then the condition $U\left(\left\{d_{f}\right\}, d_{g}\right)$ implies the uniform continuity of $g$ : let $\epsilon>0$ and $\delta>0$ be such that $U\left(d_{f}, \delta, d_{g}, \epsilon\right)$. If $x, y \in X$ then

$$
\rho(x, y) \leq \omega_{f}(\delta) \rightarrow|f(x)-f(y)| \leq \delta \rightarrow|g(x)-g(y)| \leq \epsilon
$$

ie $\omega_{g}(\epsilon)=\omega_{f}(\delta)$.

Definition 2.4 A subset $D$ of $\mathbb{D}(X)$ is a uniformity $D$ on $X$ if

$$
\begin{aligned}
& \left(D_{0}\right) \overline{0}_{X \times X} \in D, \\
& \left(D_{1}\right) d_{1}, d_{2} \in D \rightarrow d_{1} \vee d_{2} \in D, \text { and } \\
& \left(D_{2}\right) e \in \mathbb{D}(X) \rightarrow U(D, e) \rightarrow e \in D .
\end{aligned}
$$

A uniform space is a pair $\mathcal{D}=(X, D)$, where $D$ is a uniformity on $X$. A uniformity $D$ on $X$, or a uniform space $\mathcal{D}$, are called bounded if $D \subseteq \mathbb{D}^{*}(X)$. 
Clearly, $\left\{\overline{0}_{X \times X}\right\}$ and $\mathbb{D}(X)$ are uniformities on $X$ that we call the trivial and the discrete uniformity on $X$, respectively. If $D$ is a uniformity on $X$ then $\{\overline{0}\} \subseteq D \subseteq \mathbb{D}(X)$. It is immediate to see that if $D_{1}, D_{2}$ are uniformities on $X$, then $D_{1} \cap D_{2}$ is a uniformity on $X$. The next proposition expresses the independence of $\left(D_{1}\right)$ and $\left(D_{2}\right)$.

Proposition 2.5 (i) There exists a $\vee$-closed, not pseudometrically closed $\Delta \subseteq$ $\mathbb{D}(X)$.

(ii) There exist $X$ and a pseudometrically closed $\Delta \subseteq \mathbb{D}(X)$ that is not $\vee$-closed.

Proof (i) Let $\Delta=\{d\}$, where $d$ is a non-zero pseudometric on $X$. Since $d \vee d=d$, $\Delta$ is $\vee$-closed, and since $U\left(d, \frac{\epsilon}{2}, d+d, \epsilon\right)$, for every $\epsilon>0$, we get $d+d \in \bar{\Delta} \backslash \Delta$.

(ii) Let $X=\left\{x_{1}, x_{2}, x_{3}\right\}$ be a set with three elements. Let $f, g: X \rightarrow \mathbb{R}$ such that $f\left(x_{1}\right)=f\left(x_{2}\right),\left|g\left(x_{1}\right)-g\left(x_{2}\right)\right|=\epsilon_{12}>0$, and $g\left(x_{1}\right)=g\left(x_{3}\right),\left|f\left(x_{1}\right)-f\left(x_{3}\right)\right|=\epsilon_{13}>0$. By definition

$$
d_{f} \vee d_{g} \in \overline{\left\{d_{f}, d_{g}\right\}} \leftrightarrow \forall_{\epsilon>0} \exists_{\delta>0}\left(U\left(d_{f}, \delta, d_{f} \vee d_{g}, \epsilon\right) \vee U\left(d_{g}, \delta, d_{f} \vee d_{g}, \epsilon\right)\right) .
$$

We suppose that $d_{f} \vee d_{g} \in \overline{\left\{d_{f}, d_{g}\right\}}$ and we apply the above condition on some $\epsilon>0$ such that $\epsilon<\epsilon_{12} \wedge \epsilon_{13}$. If $U\left(d_{f}, \delta, d_{f} \vee d_{g}, \epsilon\right)$ is the case, then for $x_{1}, x_{2}$ we have that $\left|f\left(x_{1}\right)-f\left(x_{2}\right)\right|=0 \leq \delta$ and $\left|f\left(x_{1}\right)-f\left(x_{2}\right)\right| \vee\left|g\left(x_{1}\right)-g\left(x_{2}\right)\right|=\epsilon_{12} \leq \epsilon$, which is a contradiction. If $U\left(d_{g}, \delta, d_{f} \vee d_{g}, \epsilon\right)$ is the case, then for $x_{1}, x_{3}$ we have that $\left|g\left(x_{1}\right)-g\left(x_{3}\right)\right|=0 \leq \delta$ and $\left|f\left(x_{1}\right)-f\left(x_{3}\right)\right| \vee\left|g\left(x_{1}\right)-g\left(x_{3}\right)\right|=\epsilon_{13} \leq \epsilon$, which is a contradiction. Hence $d_{f} \vee d_{g} \notin \overline{\left\{d_{f}, d_{g}\right\}}$.

Definition 2.6 If $d \in \mathbb{D}(X)$ and $a>0$, the truncation of $d$ by $a$ is the mapping $d \wedge \bar{a}_{X \times X}$, where $\left(d \wedge \bar{a}_{X \times X}\right)(x, y)=d(x, y) \wedge \bar{a}_{X \times X}(x, y)=d(x, y) \wedge a$ for every $x, y \in X$, and $a \wedge b:=\min \{a, b\}$ for every $a, b \in \mathbb{R}$.

Proposition 2.7 If $\mathcal{D}=(X, D)$ is a uniform space and $e \in \mathbb{D}(X)$, the following hold.

$\left(D_{3}\right) a>0 \rightarrow d \in D \rightarrow \bar{a}_{X \times X} d \in D$.

$\left(D_{4}\right) e \leq d \rightarrow d \in D \rightarrow e \in D$.

$\left(D_{5}\right) \mathrm{D}$ is inhabited.

(D) $d_{1}, d_{2} \in D \rightarrow d_{1}+d_{2} \in D$.

$\left(D_{7}\right) a>0 \rightarrow d \in D \rightarrow d \wedge \bar{a}_{X \times X} \in D$.

$\left(D_{8}\right)$ If $d \in D$ and $x_{0} \in X$, the pseudometric $d_{d_{x_{0}}}$ on $X$ induced by $d_{x_{0}}$ is in $D$.

Proof $\quad\left(D_{3}\right)$ If $\epsilon>0, x, y \in X$, and $d(x, y) \leq \frac{\epsilon}{a}$, then $\left(\bar{a}_{X \times X} d\right)(x, y) \leq \epsilon$, ie $\bar{a}_{X \times X} d \in \overline{\{d\}} \subseteq \bar{D}=D$. 
$\left(D_{4}\right)$ If $\epsilon>0$ and $x, y \in X$, then if $d(x, y) \leq \epsilon$ then $e(x, y) \leq \epsilon$, ie $e \in \overline{\{d\}} \subseteq \bar{D}=D$.

$\left(D_{5}\right)$ Immediate by $\left(D_{0}\right)$.

$\left(D_{6}\right)$ Since $d_{1}, d_{2} \leq d_{1} \vee d_{2}, d_{1}+d_{2} \leq \overline{2}_{X \times X}\left(d_{1} \vee d_{2}\right)$, and we use $\left(D_{3}\right)$ and $\left(D_{4}\right)$.

$\left(D_{7}\right)$ The triangle inequality, the only nontrivial condition in showing $d \wedge \bar{a}_{X \times X} \in \mathbb{D}(X)$, follows from the property $(b+c) \wedge a=(b \wedge a)+(c \wedge a)$ of reals. If $x, y \in X$ and $\epsilon>0$ then $d(x, y) \leq \frac{a}{2} \wedge \epsilon \rightarrow\left(d \wedge \bar{a}_{X \times X}\right)(x, y)=d(x, y) \leq \epsilon$, and we use $\left(D_{2}\right)$.

$\left(D_{8}\right)$ If $x_{1}, x_{2} \in X$, we have that

$$
d_{d_{x_{0}}}\left(x_{1}, x_{2}\right)=\left|d_{x_{0}}\left(x_{1}\right)-d_{x_{0}}\left(x_{2}\right)\right|=\left|d\left(x_{1}, x_{0}\right)-d\left(x_{2}, x_{0}\right)\right| \leq d\left(x_{1}, x_{2}\right)
$$

therefore $d_{d_{x_{0}}} \leq d$. By $\left(D_{4}\right)$ we get $d_{d_{x_{0}}} \in D$.

Since $d_{1} \vee d_{2} \leq d_{1}+d_{2}$, one could replace $\left(D_{1}\right)$ with $\left(D_{6}\right)$. Moreover, $\left(D_{0}\right)$ is equivalent to $\left(D_{5}\right)$. One can turn the definitional clauses $\left(D_{0}\right),\left(D_{1}\right)$ and $\left(D_{2}\right)$ of a uniformity into inductive rules and define the least uniformity generated by some given set of pseudometrics $D_{0}$. This notion is central to the development of the constructive study of uniformities of pseudometrics. ${ }^{7}$

Definition 2.8 If $D_{0} \subseteq \mathbb{D}(X)$, the least uniformity $\coprod D_{0}$ generated by $D_{0}$, is defined by the following inductive rules:

$$
\begin{gathered}
\frac{d_{0} \in D_{0}}{d_{0} \in \coprod D_{0}} \quad \overline{\overline{0}_{X \times X} \in \coprod D_{0}} \\
\frac{d_{1}, d_{2} \in \coprod D_{0}}{d_{1} \vee d_{2} \in \coprod D_{0}} \\
\frac{\left(d \in \coprod D_{0} \wedge \delta>0 \wedge U(d, \delta, e, \epsilon)\right)_{\epsilon>0}}{e \in \coprod D_{0}}
\end{gathered}
$$

If $D$ is a uniformity on $X, D_{0} \subseteq \mathbb{D}(X)$, and $D=\coprod D_{0}$, we call $D_{0}$ a subbase for $D$.

The most complex inductive rule in Definition 2.8 can be replaced by the following rule

$$
\frac{d_{1} \in \coprod D_{0} \wedge \delta_{1}>0 \wedge U\left(d_{1}, \delta_{1}, e, 1\right), d_{2} \in \coprod D_{0} \wedge \delta_{2}>0 \wedge U\left(d_{2}, \delta_{2}, e, \frac{1}{2}\right), \ldots}{e \in \coprod D_{0}}
$$

\footnotetext{
${ }^{7}$ In the constructive theory of uniformities of entourages (see Bridges and Vîţă [13]), such a notion cannot be defined.
} 
which has countably many premisses. Definition 2.8 induces the following induction principle Ind $\amalg D_{0}$ on $\bigsqcup D_{0}$. If $P$ is any property on $\mathbb{D}(X)$, then:

$$
\begin{aligned}
& \forall_{d_{0} \in D_{0}}\left(P\left(d_{0}\right)\right) \rightarrow \\
& P\left(\overline{0}_{X \times X}\right) \rightarrow \\
& \forall_{d_{1}, d_{2} \in \amalg D_{0}}\left(P\left(d_{1}\right) \rightarrow P\left(d_{2}\right) \rightarrow P\left(d_{1} \vee d_{2}\right)\right) \rightarrow \\
& \forall_{e \in \amalg D_{0}}\left(\forall_{\epsilon>0} \exists_{\delta>0} \exists_{d \in \amalg D_{0}}(P(d) \wedge U(d, \delta, e, \epsilon)) \rightarrow P(e)\right) \rightarrow \\
& \forall_{d \in \amalg D_{0}}(P(d))
\end{aligned}
$$

Definition 2.9 A property $P$ on $\mathbb{D}(X)$ is $\amalg$-lifted if

$$
\forall_{d_{0} \in D_{0}}\left(P\left(d_{0}\right) \rightarrow \forall_{d \in \amalg D_{0}}(P(d)),\right.
$$

while it is lifted to the closure if for every $D_{0} \subseteq \mathbb{D}(X)$,

$$
\forall_{d_{0} \in D_{0}}\left(P\left(d_{0}\right) \rightarrow \forall_{d \in \overline{D_{0}}}(P(d)) .\right.
$$

Definition 2.10 If $D$ is a uniformity on $X, \Delta \subseteq D$ is called a base for $D$ if $D \subseteq \bar{\Delta}$.

Since $\Delta \subseteq D \rightarrow \bar{\Delta} \subseteq \bar{D}=D$, we get that $\Delta$ is a base for $D$ if and only if $D=\bar{\Delta}$. Note that since $D$ is inhabited, a base $\Delta$ for $D$ is also inhabited, while a subbase need not be inhabited, eg $\amalg \emptyset=\left\{\overline{0}_{X \times X}\right\}$. The following two propositions are easy to show.

Proposition 2.11 If $\Delta \subseteq \mathbb{D}(X)$ is $\vee$-closed then $\bar{\Delta}$ is $\vee$-closed.

Proposition 2.12 Let $(X, D)$ be a uniform space and $D_{0}$ an inhabited subbase for $D$.

(i) If $D_{0}$ is $\vee$-closed then $D_{0}$ is a base for $\amalg D_{0}$.

(ii) The set $\Delta\left(D_{0}\right)=\left\{\bigvee_{i=1}^{n} d_{0 i} \mid d_{0 i} \in D_{0}, 1 \leq i \leq n, n \in \mathbb{N}\right\}$ is a base for $D .{ }^{8}$

(iii) The set of bounded pseudometrics $D^{*}=D \cap \mathbb{D}^{*}(X)$ of $D$ is a base for $D$.

(iv) If $\Delta$ is a base for $D$ and $a>0, \Delta \wedge \bar{a}_{X \times X}=\left\{d \wedge \bar{a}_{X \times X} \mid d \in \Delta\right\}$ is a base for $D$.

By Proposition 2.12(iii), although $D^{*}$ contains the zero pseudometric on $X$ and it is $V$ closed, it is not in general pseudometrically closed, since if it was, every uniformity on $X$ would be bounded, which of course is not the case. The fact that $D^{*}$ is not a uniformity reveals a difference between the notion of a Bishop topology, where the bounded elements $F^{*}$ of a Bishop topology $F$ form a Bishop topology (see Definition 4.2), and the notion of uniformity of pseudometrics.

\footnotetext{
${ }^{8}$ The generation of a base out of a subbase for Bishop spaces is more complex; see Petrakis [33].
} 
Definition 2.13 If $\mathcal{D}=(X, D), \mathcal{E}=(Y, E)$ are uniform spaces, a function $h: X \rightarrow Y$ is a morphism from $\mathcal{D}$ to $\mathcal{E}$ if and only if $\forall_{e \in E}(e \odot h \in D)$, where the pseudo-composition operation $\odot$ of the pseudometric $e$ and the function $h$ is defined $^{9}$ by

$$
\begin{aligned}
& e \odot h:=e \circ h^{[2]} \\
& h^{[2]}\left(x_{1}, x_{2}\right):=\left(h\left(x_{1}\right), h\left(x_{2}\right)\right)
\end{aligned}
$$

for every $x_{1}, x_{2} \in X$, ie the following diagram commutes:

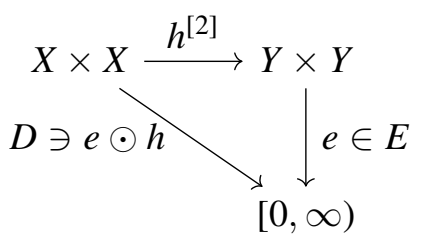

We denote by $\operatorname{Mor}(\mathcal{D}, \mathcal{E})$ the morphisms between $\mathcal{D}$ and $\mathcal{E}$. If $h \in \operatorname{Mor}(\mathcal{D}, \mathcal{E})$, it is called open, if $\forall_{d \in D} \exists_{e \in E}(d=e \odot h)$, an isomorphism between $\mathcal{D}$ and $\mathcal{E}$, if it is a bijection and $h^{-1} \in \operatorname{Mor}(\mathcal{E}, \mathcal{D})$, and a set-epimorphism, if $h$ is a surjection, while the induced mapping $H$ of $h$ is the function $H: E \rightarrow D$, defined by $H(e):=e \odot h$, for every $e \in E$. The morphisms between uniform spaces are the arrows in the category of uniform spaces Unif, where the identity arrow for $\mathcal{D}$ is the identity function $\operatorname{id}_{X}$ of $X$.

The proof of the next proposition is straightforward.

Proposition 2.14 Suppose that $\mathcal{D}=(X, D), \mathcal{E}=(Y, E), \mathcal{B}=(Z, B)$ are uniform spaces, $h \in \operatorname{Mor}(\mathcal{D}, \mathcal{E}), e_{1}, e_{2} \in E, \epsilon, \delta>0, Z \subseteq E$, and $Z \odot h:=\{\zeta \odot h \mid \zeta \in Z\}$.

(i) $\overline{0}_{Y \times Y} \odot h=\overline{0}_{X \times X}$.

(ii) $\left(e_{1} \vee e_{2}\right) \odot h=\left(e_{1} \odot h\right) \vee\left(e_{2} \odot h\right)$.

(iii) $\left(e_{1}+e_{2}\right) \odot h=\left(e_{1} \odot h\right)+\left(e_{2} \odot h\right)$.

(iv) $U\left(e_{1}, \delta, e_{2}, \epsilon\right) \rightarrow U\left(e_{1} \odot h, \delta, e_{2} \odot h, \epsilon\right)$.

(v) If $e \in \bar{Z}$, then $e \odot h \in \overline{Z \odot h}$.

(vi) If $h$ is a set-epimorphism, then $h$ is an isomorphism if and only if $h$ is open.

(vii) If $g \in \operatorname{Mor}(\mathcal{E}, \mathcal{B})$, then $(b \odot g) \odot h=b \odot(g \circ h)$, for every $b \in B$.

(viii) If $h$ is a set-epimorphism and $e_{1} \odot h=e_{2} \odot h$, then $e_{1}=e_{2}$.

\footnotetext{
${ }^{9}$ It is immediate to see that $e \odot h$ is a pseudometric on $X$.
} 
If $(X, T)$ is a topological space, the set $C(X)$ of real-valued continuous functions on $X$ is a ring and a lattice. To this structure of $C(X)$ corresponds the notion of a ring and lattice homomorphism. The algebraic and lattice structure of a uniformity $D$ on some $X$ can be described by the signature

$$
\left(\overline{0}_{X \times X}, \vee,+,\left(U_{\epsilon, \delta}\right)_{\epsilon, \delta>0}\right)
$$

where $\left(D, \overline{0}_{X \times X}, \vee\right)$ is a semi-lattice with bottom, $\left(D,+, \overline{0}_{X \times X}\right)$ is an abelian monoid with unit, and for every $\epsilon, \delta>0$ the relation $U_{\epsilon, \delta} \subseteq D \times D$ is defined by $U_{\epsilon, \delta}(d, e)=$ $U(d, \delta, e, \epsilon)$. To this structure of $D$ corresponds a natural notion of homomorphism. By Proposition 2.14 the induced mapping $H$ of some $h \in \operatorname{Mor}(\mathcal{D}, \mathcal{E})$ is such a homomorphism.

Definition 2.15 If $\mathcal{D}=(X, D), \mathcal{E}=(Y, E)$ are uniform spaces, a function $\Phi: D \rightarrow E$ is called a uniformity homomorphism if it preserves $\overline{0}_{X \times X}, \vee,+$, and

$$
U_{\epsilon, \delta}\left(d_{1}, d_{2}\right) \rightarrow U_{\epsilon, \delta}\left(\Phi\left(d_{1}\right), \Phi\left(d_{2}\right)\right)
$$

for every $d_{1}, d_{2} \in D$ and $\delta, \epsilon>0$. If $\Phi$ is a bijection and $\Phi^{-1}$ is a uniformity homomorphism, then $\Phi$ is called a uniformity isomorphism.

Proposition 2.16 ( $\left(\right.$-lifting of morphisms) If $\mathcal{D}=(X, D)$ and $\mathcal{E}=\left(Y, \coprod E_{0}\right)$ are uniform spaces, then $h: X \rightarrow Y \in \operatorname{Mor}(\mathcal{D}, \mathcal{E})$ if and only if $\forall_{e_{0} \in E_{0}}\left(e_{0} \odot h \in D\right)$ :

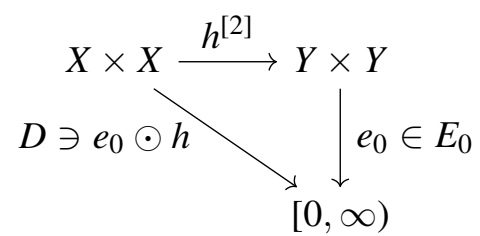

Proof We show inductively that $\forall_{e \in \coprod E_{0}}(e \odot h \in D)$. This is immediate if $e \in E_{0}$ or $e=\overline{0}_{Y \times Y}$. For the case $e_{1} \vee e_{2}$ we use Proposition 2.14(ii) and the inductive hypotheses on $e_{1}$ and $e_{2}$. If $\epsilon, \delta>0$, the property $U\left(e^{\prime}, \delta, e, \epsilon\right) \rightarrow U\left(e^{\prime} \odot h, \delta, e \odot h, \epsilon\right)$, where $e^{\prime} \in \amalg E_{0}$ such that $e^{\prime} \odot h \in D$, is shown by Proposition 2.14(iv).

Lemma 2.17 (Well-definability lemma) Let $h: X \rightarrow Y$ be a surjection, $Z \subseteq \mathbb{D}(Y)$, and $d \in \mathbb{D}(X)$. If $d \in \overline{Z \odot h}$, the function $d^{\#}: Y \times Y \rightarrow \mathbb{R}$, defined by

$$
d^{\#}\left(y_{1}, y_{2}\right)=d^{\#}\left(h\left(x_{1}\right), h\left(x_{2}\right)\right):=d\left(x_{1}, x_{2}\right)
$$




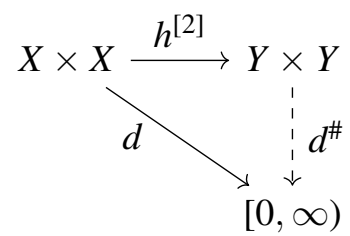

for every $y_{1}, y_{2} \in Y$, is a well-defined pseudometric on $Y$, ie

$$
\forall_{x_{1}, x_{2}, x_{3}, x_{4} \in X}\left(h\left(x_{1}\right)=h\left(x_{3}\right) \rightarrow h\left(x_{2}\right)=h\left(x_{4}\right) \rightarrow d\left(x_{1}, x_{2}\right)=d\left(x_{3}, x_{4}\right)\right) .
$$

Proof Let $x_{1}, x_{2}, x_{3}, x_{4} \in X$ with $h\left(x_{1}\right)=h\left(x_{3}\right)=y_{1}$ and $h\left(x_{2}\right)=h\left(x_{4}\right)=y_{2}$. Since $d \in \overline{Z \odot h}$, for every $\epsilon>0$ there are $\delta>0$ and $\zeta \in Z$ such that $U(\zeta \odot h, \delta, d, \epsilon)$, ie

$$
\forall_{x, x^{\prime} \in X}\left(\zeta\left(h(x), h\left(x^{\prime}\right)\right) \leq \delta \rightarrow d\left(x, x^{\prime}\right) \leq \epsilon\right) .
$$

Let $\epsilon>0$. There exist $\delta>0$ and $\zeta \in Z$ such that $\forall_{x, x^{\prime} \in X}\left(\zeta\left(h(x), h\left(x^{\prime}\right)\right) \leq \delta \rightarrow\right.$ $\left.d\left(x, x^{\prime}\right) \leq \frac{\epsilon}{2}\right)$. Since by hypothesis $\zeta\left(h\left(x_{1}\right), h\left(x_{3}\right)\right)=\zeta\left(h\left(x_{2}\right), h\left(x_{4}\right)\right)=0$, we get $d\left(x_{1}, x_{3}\right) \leq \frac{\epsilon}{2}$ and $d\left(x_{2}, x_{4}\right) \leq \frac{\epsilon}{2}$. Hence

$$
\left|d\left(x_{1}, x_{2}\right)-d\left(x_{3}, x_{4}\right)\right| \leq d\left(x_{1}, x_{3}\right)+d\left(x_{2}, x_{4}\right) \leq \frac{\epsilon}{2}+\frac{\epsilon}{2}=\epsilon .
$$

Since $\epsilon>0$ is arbitrary, we get $\left|d\left(x_{1}, x_{2}\right)-d\left(x_{3}, x_{4}\right)\right|=0$. That $d^{\#}$ is a pseudometric on $Y$ follows by the fact that $d$ is a pseudometric on $X$ and the surjectivity of $h$.

Proposition 2.18 (i) If $h: X \rightarrow Y$ is a surjection, $\Delta \subseteq \mathbb{D}(X)$, and $Z \subseteq \mathbb{D}(Y)$, then

$$
\forall_{d \in \Delta} \exists_{\zeta \in Z}(d=\zeta \odot h) \rightarrow \forall_{e \in \bar{\Delta}^{-}} \exists_{\zeta^{*} \in \bar{Z}}\left(e=\zeta^{*} \odot h\right) .
$$

(ii) Let $\mathcal{D}=(X, D), \mathcal{E}=(Y, E)$ be uniform spaces, $h \in \operatorname{Mor}(\mathcal{D}, \mathcal{E})$ a surjection, and let $\Delta$ be a base for $D$.

(a) (Lifting of openness to the closure of a base) If $\forall_{d \in \Delta} \exists_{e \in E}(d=e \odot h)$ then $h$ is open and $H^{-1}(\Delta)$ is a base for $E$.

(b) (【-lifting of openness) If $D_{0}$ is a subbase for $D$ such that $\forall_{d_{0} \in D_{0}} \exists_{e \in E}\left(d_{0}=\right.$ $e \odot h)$ then $\forall_{d \in D} \exists_{e \in E}(d=e \odot h)$.

Proof (i) If $e \in \bar{\Delta}$ and $\epsilon>$, there exist $d \in \Delta$ and $\delta>0$ such that $U(d, \delta, e, \epsilon)$. Since $\Delta \subseteq Z \odot h$, we have that $e \in \bar{\Delta} \subseteq \overline{Z \odot h}$, by the well-definability lemma we have that $e^{\#} \in \mathbb{D}(Y)$ and $e=e^{\#} \odot h$. If $\zeta \in Z$ such that $d=\zeta \odot h$, then

$$
U(d, \delta, e, \epsilon) \leftrightarrow U\left(\zeta \odot h, \delta, e^{\#} \odot h, \epsilon\right) .
$$


Since $h$ is onto $Y$, we get $U\left(\zeta, \delta, e^{\#}, \epsilon\right)$. Since $\epsilon>0$ is arbitrary, $e^{\#}$ is in $\bar{Z}$, ie the required element $\zeta^{*}$ of $\bar{Z}$ is $e^{\#}$.

(ii) (a) This lifting follows from (i) for $Z=E$. If $e \in E$, then $e \odot h \in D$, and since $\Delta$ is a base for $D$, if $\epsilon>0$, there are $\delta>0, d \in \Delta$ such that $U(d, \delta, e \odot h, \epsilon)$. By hypothesis there is $e^{\prime} \in E$ with $d=e^{\prime} \odot h$, hence $U\left(e^{\prime} \odot h, \delta, e \odot h, \epsilon\right)$, and consequently $U\left(e^{\prime}, \delta, e, \epsilon\right)$. Since $e^{\prime} \in H^{-1}(\Delta)$ and $\epsilon>0$ is arbitrary, $H^{-1}(\Delta)$ is a base for $E$.

(b) This lifting follows from (a) and the fact that for every element $\bigvee_{i=1}^{n} d_{0 i}$ of the base $\Delta\left(D_{0}\right)$ for $D$ (Proposition 2.12)(ii) there exist $e_{1}, \ldots, e_{n} \in E$ such that:

$$
\bigvee_{i=1}^{n} d_{0 i}=\bigvee_{i=1}^{n}\left(e_{i} \odot h\right)=\left(\bigvee_{i=1}^{n} e_{i}\right) \odot h
$$

The $\amalg$-lifting of openness is used in the proof of Theorems 3.15 and 6.6.

Definition 2.19 If $\mathcal{D}=(X, D)$ and $\mathcal{E}=(Y, E)$ are uniform spaces, the product uniform space is the pair $\mathcal{D} \times \mathcal{E}=(X \times Y, D \times E)$, where

$$
D \times E:=\coprod\left[\left\{d \odot \pi_{1} \mid d \in D\right\} \cup\left\{e \odot \pi_{2} \mid e \in E\right\}\right]=: \coprod_{d \in D}^{e \in E} d \odot \pi_{1}, e \odot \pi_{2},
$$

$\pi_{1}$ is the projection map of $X \times Y$ on $X$ and $\pi_{2}$ is the projection map of $X \times Y$ on $Y$. If $A \subseteq X$ is inhabited, the relative uniform space on $A$ is the pair $\mathcal{D}_{\mid A}=\left(A, D_{\mid A}\right)$, where:

$$
D_{\mid A}:=\coprod\left\{d_{\mid A \times A} \mid d \in D\right\}=: \coprod_{d \in D} d_{\mid A \times A}
$$

An isomorphism $h$ between $\mathcal{D}$ and $\mathcal{E}_{\mid h(X)}$ is called a uniform embedding of $\mathcal{D}$ into $\mathcal{E}$.

According to Beeson [2, page 44], if $A$ is a rule which associates to every element $i$ of a set $I$ a set $A_{i}$, the infinite product $\prod_{i \in I} A_{i}$ is defined by

$$
\prod_{i \in I} A_{i}:=\left\{f \in \mathbb{F}\left(I, \bigcup_{i \in I} A(i)\right) \mid \forall_{i \in I}(f(i) \in A(i))\right\}
$$

where the exterior union $\bigcup_{i \in I} A_{i}$ is defined by Richman (see Example 2 in [7, page 78]). If $A$ associates to every element of $I$ the set $X$, we denote the product $\prod_{i \in I} X$ by $X^{I}$. Since $X^{I}=\mathbb{F}(I, X)$, the exterior union is avoided in this case.

Definition 2.20 If $\mathbb{X}$ is a rule which associates to every element $i$ of a set $I$ a setoid $\left(X_{i},=_{i}\right)$, and $\mathbb{D}$ is a rule which associates to every element $i$ of $I$ a set $D_{i} \subseteq \mathbb{D}\left(X_{i}\right)$ such that $\mathcal{D}_{i}=\left(X_{i}, D_{i}\right)$ is a uniform space, the I-product of the uniform spaces $\mathcal{D}_{i}$ is the pair

$$
\prod_{i \in I} \mathcal{D}_{i}:=\left(\prod_{i \in I} X_{i}, \coprod_{d \in D_{i}}^{i \in I} d \odot \varpi_{i}\right)
$$


where $\varpi_{i}$ is the $i$-th projection function from $\prod_{i \in I} X_{i}$ to $X_{i}$, ie $\varpi_{i}(f)=f(i)$ for every $f \in \prod_{i \in I} X_{i}$ and every $i \in I$. If $\mathcal{D}=(X, D)$ is a uniform space and to each element of $I$ the sets $X$ and $D$ are associated, we denote the $I$-product of $\mathcal{D}$ by

$$
\mathcal{D}^{I}:=\left(X^{I}, \coprod_{d \in D}^{i \in I} d \odot \varpi_{i}\right) .
$$

It is easy to see that $D \times E$ is the least uniformity on $X \times Y$ such that $\pi_{1}, \pi_{2}$ are in $\operatorname{Mor}(\mathcal{D} \times \mathcal{E}, \mathcal{D})$ and in $\operatorname{Mor}(\mathcal{D} \times \mathcal{E}, \mathcal{E})$, respectively, and that $\mathcal{D} \times \mathcal{E}$ satisfies the universal property of the product. The following two propositions are easy to show.

Proposition 2.21 If $D_{0} \subseteq \mathbb{D}(X)$ and $E_{0} \subseteq \mathbb{D}(Y)$, and $A \subseteq X$ inhabited, then:

$$
\begin{gathered}
\coprod D_{0} \times \coprod E_{0}=\coprod\left[\left\{d_{0} \odot \pi_{1} \mid d_{0} \in D_{0}\right\} \cup\left\{e_{0} \odot \pi_{2} \mid e_{0} \in E_{0}\right\}\right] \\
=: \coprod_{d_{0} \in D_{0}} d_{0} \odot \pi_{1}, e_{0} \odot \pi_{2} \\
\left(\coprod D_{0}\right)_{\mid A}=\coprod\left\{d_{0 \mid A \times A} \mid d_{0} \in D_{0}\right\}=: \coprod_{d_{0} \in D_{0}} d_{0 \mid A \times A}
\end{gathered}
$$

Proposition 2.22 Let $\mathcal{D}=(X, D)$ be a uniform space, $x_{0} \in X$, and $d \in \mathbb{D}(X)$.

(i) The mappings ${ }_{x_{0}} i: X \rightarrow X \times X$ and $i_{x_{0}}: X \rightarrow X \times X$, defined by $x \mapsto\left(x, x_{0}\right)$ and $x \mapsto\left(x_{0}, x\right)$ for every $x \in X$, respectively, are uniform embeddings of $\mathcal{D}$ into $\mathcal{D} \times \mathcal{D}$.

(ii) If $d \odot \pi_{1} \in D \times D$, or if $d \odot \pi_{2} \in D \times D$, then $d \in D$.

It is easy to see that the previous equalities hold for the $I$-product of uniform spaces too. The next fact is also immediate to show.

Proposition 2.23 Let $\mathcal{D}=(X, D)$ and $\mathcal{E}=(Y, E)$ be uniform spaces and $h: X \rightarrow Y$.

(i) $h \in \operatorname{Mor}(\mathcal{D}, \mathcal{E})$ if and only if $h \in \operatorname{Mor}\left(\mathcal{D}, \mathcal{E}_{h(X)}\right)$.

(ii) $h$ is an open morphism from $\mathcal{D}$ to $\mathcal{E}, h$ is open as a morphism from $\mathcal{D}$ to $\mathcal{E}_{\mid h(X)}$.

If $h \in \operatorname{Mor}\left(\mathcal{D}, \mathcal{E}_{\mid h(X)}\right)$ is open, it is not necessarily open as an element of $\operatorname{Mor}(\mathcal{D}, \mathcal{E})$; if $d \in D$ and $e^{\prime} \in E_{\mid h(X)}$ with $d=e^{\prime} \odot h$, it is not necessary that $e^{\prime}=e_{\mid h(X) \times h(X)}$ for some $e \in E$. 
Definition 2.24 If $(X, \rho)$ is a metric space, we call the uniformity

$$
D(\rho)=\coprod\{\rho\}:=\coprod \rho
$$

on $X$ the metric uniformity on $X$ generated by $\rho$, and $\mathcal{D}(\rho)=(X, D(\rho))$ the metric uniform space generated by $\rho$. The uniform space

$$
\mathcal{R}=\left(\mathbb{R}, D\left(d_{\mathbb{R}}\right)\right)
$$

is the uniform space of reals. An $I$-product $\mathcal{R}^{I}$ of $\mathcal{R}$ is called a Euclidean uniform space.

If $I=n:=\{1, \ldots, n\}$, then by Proposition 2.21 we have that

$$
D\left(d_{\mathbb{R}}\right)^{n}=\left(\coprod d_{\mathbb{R}}\right)^{n}=\coprod d_{\mathbb{R}} \odot \pi_{1}, \ldots, d_{\mathbb{R}} \odot \pi_{n}=\coprod d_{\pi_{1}}, \ldots, d_{\pi_{n}}
$$

since $d_{\mathbb{R}} \odot \pi_{i}=d_{\pi_{i}}$, for every $i \in n$. In the classical literature, see eg Gillman and Jerison [21, page 224], and in the constructive one, see Bishop and Bridges [7, page 124], an element of $\operatorname{Mor}(\mathcal{D}, \mathcal{E})$ is called a uniformly continuous function. Because of Proposition 2.25(ii) the notion of a morphism between uniform spaces is a generalization of a uniformly continuous function between metric spaces. As we show though in Theorem 6.9, the notion of morphism between uniform spaces can also be reduced to other notions of continuity, like Bishop continuity. The next proposition has an immediate proof.

Proposition 2.25 Let $(X, \rho),(Y, \sigma)$ be metric spaces and $h: X \rightarrow Y$.

(i) $\amalg \rho=\overline{\{\rho\}}$.

(ii) $h \in \operatorname{Mor}(\mathcal{D}(\rho), \mathcal{D}(\sigma))$ if and only if $h$ is uniformly continuous.

Definition 2.26 If $\mathcal{D}=(X, D)$ is a uniform space, we denote by $\mathcal{M}(\mathcal{D})$ the set $\operatorname{Mor}(\mathcal{D}, \mathcal{R})$ and by $\mathcal{M}^{*}(\mathcal{D})$ the bounded elements of $\operatorname{Mor}(\mathcal{D}, \mathcal{R})$.

The next fact follows easily.

Proposition 2.27 Let $\mathcal{D}=(X, D)$ be a uniform space and $h: X \rightarrow \mathbb{R}$.

(i) $h \in \mathcal{M}(\mathcal{D}) \leftrightarrow d_{h} \in D$.

(ii) $\mathcal{D}$ is bounded if and only if $\mathcal{M}(\mathcal{D})=\mathcal{M}^{*}(\mathcal{D})$.

By Proposition 2.27(i), the fact that $d_{\bar{a}_{X}}=\overline{0}_{X \times X}$, for (3), and ( $\left.D_{8}\right)$, for (4), we get:

(1) $\left\{h \in \mathbb{F}(X) \mid d_{h} \in D\right\}$ 
(2) $\mathcal{M}^{*}(\mathcal{D})=\left\{h \in \mathbb{F}^{*}(X) \mid d_{h} \in D\right\}$

(3) $\operatorname{Const}(X \times X) \subseteq \mathcal{M}^{*}(\mathcal{D})$

(4) $\left\{d_{x} \mid d \in D, x \in X\right\} \subseteq \mathcal{M}(\mathcal{D})$

The next result, which is found as an exercise in [21, page 237] and is included here for the sake of completeness, has its analogue in the theory of Bishop spaces, namely that a Bishop topology is the set of Bishop morphisms from the Bishop space to the Bishop space of reals (see the footnote in Definition 4.2). Its proof is straightforward.

Proposition 2.28 Let $\mathcal{D}=(X, D)$ be a uniform space and $e \in \mathbb{D}(X)$.

(i) $e \in D$ if and only if $e \in \mathcal{M}(\mathcal{D} \times \mathcal{D})$.

(ii) $e \in D^{*}$ if and only if $e \in \mathcal{M}^{*}(\mathcal{D} \times \mathcal{D})$.

The hypothesis $e \in \mathbb{D}(X)$ in the formulation of Proposition 2.28 is used in the proof of both implications of case (i); it is also necessary, since the constant maps are in $\mathcal{M}^{*}(\mathcal{D} \times \mathcal{D})$ but, except from $\overline{0}_{X \times X}$, they don't satisfy the properties of a pseudometric.

Definition 2.29 If $h: X \times X \rightarrow \mathbb{R}$ and $x_{0} \in X$, we define the functions $h^{\Delta}$, $h_{x_{0}}, x_{0} h: X \rightarrow \mathbb{R}$, by $h^{\Delta}(x):=h(x, x), h_{x_{0}}(x):=h\left(x_{0}, x\right), x_{0} h(x):=h\left(x, x_{0}\right)$ for every $x \in X$, respectively. If $g: X \rightarrow Y$, we define $g^{[2]}: X \times X \rightarrow Y \times Y$ by $g^{[2]}\left(x_{1}, x_{2}\right)=\left(g\left(x_{1}\right), g\left(x_{2}\right)\right)$ for every $x_{1}, x_{2} \in X$.

The next proposition follows easily.

Proposition 2.30 Let $\mathcal{D}=(X, D), \mathcal{E}=(Y, E)$ be uniform spaces and $x_{0} \in X$.

(i) If $h: X \times X \rightarrow \mathbb{R}$ such that $h \in \mathcal{M}(\mathcal{D} \times \mathcal{D})$, the maps $h^{\Delta}, h_{x_{0}}$ and ${ }_{x_{0}} h$ are in $\mathcal{M}(\mathcal{D})$.

(ii) If $g: X \rightarrow Y$, then $g \in \operatorname{Mor}(\mathcal{D}, \mathcal{E})$ if and only if $g^{[2]} \in \operatorname{Mor}\left(\mathcal{D}^{2}, \mathcal{E}^{2}\right)$.

\section{Separating uniformities}

Definition 3.1 If $D$ is a uniformity on $X$, its canonical point-point apartness relation $\bowtie_{D}$ on $X$ is defined for every $x, y \in X$ by

$$
x \bowtie_{D} y:=\exists_{d \in D}(d(x, y)>0) .
$$


If $\bowtie_{D}$ is tight, ${ }^{10}$ then $D$ is called a tight uniformity. A subset $\Delta$ of $\mathbb{D}(X)$ is a separating set of pseudometrics, if

$$
\forall_{x, y \in X}\left(\forall_{d \in \Delta}(d(x, y)=0) \rightarrow x=y\right) .
$$

If $D$ is separating, we call $\mathcal{D}$ separated by $D$, or simply separated.

Next characterization of tightness follows immediately, while the easy to show Proposition 3.3 implies that a metric uniformity $D(\rho)$ is separating.

Proposition 3.2 If $D$ is a uniformity on $X$, then $D$ is tight if and only $D$ is separating.

Proposition 3.3 If $D_{0}$ is a subbase of a uniformity $D$ on $X$, then $D$ is separating if and only if $\forall_{x, y \in X}\left(\forall_{d_{0} \in D_{0}}\left(d_{0}(x, y)=0\right) \rightarrow x=y\right)$.

Corollary 3.4 If $(\mathcal{D}=(X, D)$ is a uniform space and $\Delta$ is a base for $D$, then $D$ is separating if and only if $\Delta$ is separating.

The next proposition is also easy to show.

Proposition 3.5 Let $\mathcal{D}=(X, D)$ and $\mathcal{E}=(Y, E)$ be uniform spaces.

(i) If $h$ is an isomorphism between $\mathcal{D}, \mathcal{E}$ and $D$ is separating, then $E$ is separating.

(ii) If $A \subseteq X$ is inhabited and $D$ is separating, then $D_{\mid A}$ is separating.

(iii) $D \times E$ is separating if and only if $D, E$ are separating.

Definition 3.6 If $\mathcal{D}=(X, D)$ is a uniform space and $\phi: X \rightarrow Y$ is a surjection, the quotient uniformity $D_{\phi}$ on $Y$ with respect to $\phi$ is defined by

$$
D_{\phi}:=\{e \in \mathbb{D}(Y) \mid e \odot \phi \in D\}
$$

and the quotient uniform space with respect to $\phi$ is the pair $\mathcal{D}_{\phi}=\left(Y, D_{\phi}\right)$.

That $D_{\phi}$ is a uniformity on $Y$ is shown through Proposition 2.14(i), (ii) and (iv), since for these equalities $h$ need not be a morphism, just a function from $X$ to $Y$.

\footnotetext{
${ }^{10}$ A point-point apartness relation $\bowtie$ on $X$ is called tight if $\forall_{x, y \in X}(\neg(x \bowtie y) \rightarrow x=y)$. The equivalent formulation of the tightness of $\nwarrow_{D}$, given in Proposition 3.2, is part of Bishop's definition of an equalizing family of pseudometrics found in [6]. In the classical literature, see eg [21], the term Hausdorff uniformity is used instead. Here we use similar terms for the corresponding notions within the theory of Bishop spaces (see Definition 4.2).
} 
Proposition 3.7 Suppose that $\mathcal{D}=(X, D), \mathcal{B}=(Z, B)$ are uniform spaces, $E$ is a uniformity on $Y$, and $\phi: X \rightarrow Y$ is a surjection.

(i) $D_{\phi}$ is the largest uniformity on $Y$ with respect to which $\phi$ is a morphism.

(ii) A function $h: Y \rightarrow Z$ is in $\operatorname{Mor}\left(\mathcal{D}_{\phi}, \mathcal{B}\right)$ if and only if $h \circ \phi \in \operatorname{Mor}(\mathcal{D}, \mathcal{B})$.

(iii) If $\phi$ is an open morphism with respect to $D$ and $E$, then $E=D_{\phi}$.

Proof (i) This is immediate from the definition of a morphism between uniform spaces. (ii) By Proposition 2.14(vii) we have that:

$$
\begin{aligned}
h \in \operatorname{Mor}\left(\mathcal{D}_{\phi}, \mathcal{B}\right) & \leftrightarrow \forall_{b \in B}\left(b \odot h \in D_{\phi}\right) \\
& \leftrightarrow \forall_{b \in B}((b \odot h) \odot \phi \in D) \\
& \leftrightarrow \forall_{b \in B}(b \odot(h \circ \phi) \in D) \\
& \leftrightarrow h \circ \phi \in \operatorname{Mor}(\mathcal{D}, \mathcal{B})
\end{aligned}
$$

(iii) Since $\phi \in \operatorname{Mor}(\mathcal{D}, \mathcal{E})$, by (i) we get $E \subseteq D_{\phi}$. If $d \in D_{\phi}$, ie $d \odot \phi \in D$, then by the supposed openness of $\phi$ there is some $e \in E$ such that $d \odot \phi=e \odot \phi$. By Proposition 2.14(viii) we get $d=e$, and hence $D_{\phi} \subseteq E$.

Proposition 3.8 If $\mathcal{D}=(X, D)$ is a uniform space, we define

$$
x_{1} \sim x_{2}:=\forall_{d \in D}\left(d\left(x_{1}, x_{2}\right)=0\right)
$$

for every $x_{1}, x_{2} \in X$. Let $X / \sim$ be the set of all equivalence classes of the equivalence relation $\sim$, let $\pi: X \rightarrow X / \sim$ be the map defined by $x \mapsto[x]_{\sim}$ for every $x \in X$, and $\mathbb{D}_{\sim}=\left(X / \sim, D_{\pi}\right)$ the quotient uniform space with respect to $\pi$.

(i) For every $d \in D$, the mapping $\tilde{d}: X / \sim \times X / \sim \rightarrow \mathbb{R}$, defined by $\tilde{d}\left(\left[x_{1}\right]_{\sim},\left[x_{2}\right]_{\sim}\right)$ $=d\left(x_{1}, x_{2}\right)$ for every $x_{1}, x_{2} \in X$, is a well-defined pseudometric on $X / \sim$ that is in $D_{\pi}$.

(ii) $\pi$ is an open morphism from $\mathcal{D}$ to $\mathbb{D}_{\sim}$.

(iii) The map $\sim: D \rightarrow D_{\pi}$, defined by $d \mapsto \tilde{d}$ for every $d \in D$, is a uniformity epimorphism.

Proof (i) and (ii) If $d \in D, \tilde{d}$ is well-defined; if $x_{1}, x_{2}, x_{3}, x_{4} \in X$ with $x_{1} \sim x_{3}$ and $x_{2} \sim x_{4}$, then $\left|d\left(x_{1}, x_{2}\right)-d\left(x_{3}, x_{4}\right)\right| \leq d\left(x_{1}, x_{3}\right)+d\left(x_{2}, x_{4}\right)=0$, hence $\tilde{d}\left(\left[x_{1}\right]_{\sim},\left[x_{2}\right]_{\sim}\right)=$ $d\left(x_{1}, x_{2}\right)=d\left(x_{3}, x_{4}=\tilde{d}\left(\left[x_{3}\right]_{\sim},\left[x_{4}\right]_{\sim}\right)\right.$. The fact that $\tilde{d}$ is a pseudometric on $X / \sim$ is trivial. Since $(\tilde{d} \odot \pi)\left(x_{1}, x_{2}\right)=\tilde{d}\left(\pi\left(x_{1}\right), \pi\left(x_{2}\right)\right)=d\left(x_{1}, x_{2}\right)$ for every $x_{1}, x_{2} \in X$, we get $\tilde{d} \odot \pi=d$, therefore $\tilde{d} \in D_{\pi}$. The last equality shows that $\pi$ is an open morphism.

(iii) First we show that it is a surjection; if $e \in D_{\pi}$, ie $e \odot \pi \in D$, then $\widetilde{e \odot \pi}=e$, since $(\widetilde{e \odot \pi})\left(\left[x_{1}\right]_{\sim},\left[x_{2}\right]_{\sim}\right)=(e \odot \pi)\left(x_{1}, x_{2}\right)=e\left(\left[x_{1}\right]_{\sim},\left[x_{2}\right]_{\sim}\right)$ for every $x_{1}, x_{2} \in X$. The properties of a uniformity homomorphism follow immediately for $\sim$. 
The next result shows that it suffices to work with uniform spaces with separating uniformities. Its proof is a translation of the classical Stone-Čech theorem for topological spaces, which expresses a similar sufficiency of the completely regular topological spaces (see Walker [46, page 6]). Note that in Gillman and Jerison [21, page 219], a different result motivated the sufficiency of uniform spaces with a separating uniformity.

Theorem 3.9 (Stone-Čech theorem for uniform spaces) If $\mathcal{D}=(X, D)$ is a uniform space, there exists a uniform space $\rho \mathcal{D}=(\rho X, \rho D)$ and a mapping $\tau_{X}: X \rightarrow \rho X \in$ $\operatorname{Mor}(\mathcal{D}, \rho \mathcal{D})$ such that the following hold.

(i) The uniformity $\rho D$ is separating.

(ii) The induced mapping $T_{X}: \rho D \rightarrow D$ of $\tau_{X}$ is a uniformity isomorphism.

(iii) If $d \in D$, there is a unique $\rho d \in \rho D$ such that the following diagram commutes:

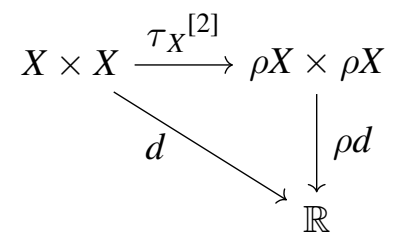

Proof Let $\rho X:=X / \sim, \rho D:=D_{\pi}$ and $\tau_{X}=\pi$, where $\sim$ is defined in Proposition 3.8.

(i) By Proposition 3.8, if $x_{1}, x_{2} \in D$, we have that

$$
\forall_{\tilde{d} \in D_{\pi}}\left(\tilde{d}\left(\left[x_{1}\right]_{\sim},\left[x_{2}\right]_{\sim}\right)=0\right) \leftrightarrow \forall_{d \in D}\left(d\left(x_{1}, x_{2}\right)=0\right) \leftrightarrow x_{1} \sim x_{2} \leftrightarrow\left[x_{1}\right]_{\sim}=\left[x_{2}\right]_{\sim} .
$$

(ii) By Proposition 3.8(iii) every element of $D_{\pi}$ is of the form $\tilde{d}$ for some $d \in D$, hence the induced mapping $\Pi$ of $\pi$ is defined by $\Pi(\tilde{d})=\tilde{d} \odot \pi=d$. The fact that $\Pi$ is a uniformity homomorphism follows immediately. Its inverse is the uniformity homomorphism $\sim$ defined in Proposition 3.8(iii), since $d \stackrel{\sim}{\mapsto} \tilde{d} \stackrel{\Pi}{\mapsto} d$ and $\tilde{d} \stackrel{\Pi}{\mapsto} d \stackrel{\sim}{\mapsto} \tilde{d}$ for every $d \in D$. We define $T_{X}=\Pi$.

(iii) It follows immediately if we define $\rho d=\tilde{d}$.

Proposition 3.10 Let $\mathcal{D}=(X, D)$ be a uniform space.

(i) If $D_{0}$ is a subbase for $D$, then $\rho D_{0}=\left\{\rho d_{0} \mid d_{0} \in D_{0}\right\}$ is a subbase for $\rho D$.

(ii) If $\Delta$ is a base $D$, then $\rho \Delta=\{\rho d \mid d \in \Delta\}$ is a base for $\rho D$.

Proof (i) We show that $\rho \coprod D_{0}=\coprod \rho D_{0}$. Since $\rho d_{0} \in \rho D$ for every $d_{0} \in D_{0}$, $\coprod \rho D_{0} \subseteq \rho D$. By a simple induction on $\coprod D_{0}$ we get $\left\{\rho d \mid d \in \coprod D_{0}\right\} \subseteq \coprod \rho D_{0}$.

(ii) If $d \in D$ and $\epsilon>0$, there are $\delta>0, d^{\prime} \in \Delta$ with $U\left(d^{\prime}, \delta, d, \epsilon\right)$. Since $\rho d^{\prime}\left(\left[x_{1}\right]_{\sim},\left[x_{2}\right]_{\sim}\right)=d^{\prime}\left(x_{1}, x_{2}\right)$ and $\rho d\left(\left[x_{1}\right]_{\sim},\left[x_{2}\right]_{\sim}\right)=d\left(x_{1}, x_{2}\right)$ for every $x_{1}, x_{2} \in X$, we get $U\left(\rho d^{\prime}, \delta, \rho d, \epsilon\right)$. 
Definition 3.11 We call $\Phi \subseteq \mathbb{F}(X)$ separating, if

$$
\forall_{x, y \in X}\left(\forall_{f \in \Phi}(f(x)=f(y)) \rightarrow x=y\right) .
$$

The set $\Phi$ induces the equivalence relation $\approx$ on $X$, defined by $x_{1} \approx x_{2}:=\forall_{f \in \Phi}(f(x)=$ $f(y))$ for every $x_{1}, x_{2} \in X$. If $f \in \Phi$, the map $\rho f: X / \approx \rightarrow \mathbb{R}$, defined by $\rho f\left([x]_{\approx}\right)=$ $f(x)$, is, by the definition of $\approx$, well-defined. Moreover, we define $\rho \Phi:=\{\rho f \mid f \in \Phi\}$.

Definition 3.12 A uniform space $\mathbb{D}=(X, D)$ is called functionally determined, or an f-uniform space, and $D$ is called an f-uniformity, if there exists $\Phi \subseteq \mathbb{F}(X)$ such that:

$$
D=\coprod_{f \in \Phi} d_{f}
$$

In this case we say that $\Phi$ determines $D$, or that $\Phi$ is a determining family for $D$. We denote by $f$-Unif the full subcategory ${ }^{11}$ of $f$-uniform spaces of Unif .

If $\Phi \subseteq \mathbb{F}(X)$ and $\sim$ is the equivalence relation on $X$ generated by the family of pseudometrics $\left\{d_{f} \mid f \in \Phi\right\}$, then for every $x_{1}, x_{2} \in X$ we have that:

$$
\begin{aligned}
x_{1} \sim x_{2} & :=\forall_{f \in \Phi}\left(d_{f}(x, y)=0\right) \\
& \leftrightarrow \forall_{f \in \Phi}(|f(x)-f(y)|=0) \\
& \leftrightarrow \forall_{f \in \Phi}(f(x)=f(y)) \\
& :=x_{1} \approx x_{2}
\end{aligned}
$$

Proposition 3.13 Let $\mathcal{D}=\left(X, \coprod_{f \in \Phi} d_{f}\right), \mathcal{E}=\left(Y, \coprod_{g \in \Theta} d_{g}\right)$ be f-uniform spaces.

(i) The product $\mathcal{D} \times \mathcal{E}$ is an $\mathrm{f}$-uniform space.

(ii) If $A \subseteq X$ is inhabited, the relative space $\mathcal{D}_{\mid A}$ is an $f$-uniform space.

(iii) $\bigsqcup_{f \in \Phi} d_{f}$ is separating if and only if $\Phi$ is separating.

Proof (i) and (ii) Since $\Phi \subseteq \mathbb{F}(X)$ and $\Theta \subseteq \mathbb{F}(Y)$, if we define the sets

$$
\begin{gathered}
\Phi \circ \pi_{1}:=\left\{f \circ \pi_{1} \mid f \in \Phi\right\} \subseteq \mathbb{F}(X \times Y) \\
\Theta \circ \pi_{2}:=\left\{g \circ \pi_{2} \mid g \in \Theta\right\} \subseteq \mathbb{F}(X \times Y) \\
\Phi_{\mid A}:=\left\{f_{\mid A} \mid f \in \Phi\right\} \subseteq \mathbb{F}(A)
\end{gathered}
$$

\footnotetext{
${ }^{11}$ For all categorical notions mentioned here we refer to Awodey [1] or Mac Lane [27].
} 
then

$$
\begin{aligned}
\left(\coprod_{f \in \Phi} d_{f}\right) \times\left(\coprod_{g \in \Theta} d_{g}\right) & =\coprod_{f \in \Phi}^{g \in \Theta} d_{f} \odot \pi_{1}, d_{g} \odot \pi_{2} \\
& =\coprod_{f \in \Phi}^{g \in \Theta} d_{f \circ \pi_{1}}, d_{g \circ \pi_{2}} \\
& =\coprod_{f^{\prime} \in \Phi \circ \pi_{1}}^{g^{\prime} \in \Theta \circ \pi_{2}} d_{f^{\prime}}, d_{g^{\prime}} \\
\left(\coprod_{f \in \Phi} d_{f}\right)_{\mid A}=\coprod_{f \in \Phi}\left(d_{f}\right)_{\mid A \times A} & =\coprod_{f \in \Phi} d_{\left(f_{\mid A}\right)}=\coprod_{f^{\prime \prime} \in \Phi_{\mid A}} d_{f^{\prime \prime}}
\end{aligned}
$$

and

(iii) By Proposition 3.3 and the equivalences $\forall_{f \in \Phi}\left(d_{f}(x, y)=0\right) \leftrightarrow \forall_{f \in \Phi}(|f(x)-f(y)|=$ 0) $\leftrightarrow \forall_{f \in \Phi}(f(x)=f(y))$.

Proposition 3.14 Let $\mathcal{D}=(X, D), \mathcal{E}=(Y, E)$ be f-uniform spaces.

(i) $\rho \mathcal{D}$ is an $\mathrm{f}$-uniform space.

(ii) $\rho(\mathcal{D} \times \mathcal{E})=\rho \mathcal{D} \times \rho \mathcal{E}$.

(iii) If $A \subseteq X$ is inhabited, then $\rho\left(\mathcal{D}_{\mid A}\right)=(\rho \mathcal{D})_{\mid A}$.

Proof Let $D=\coprod_{f \in \Phi} d_{f}$ and $E=\coprod_{g \in \Theta} d_{g}, x_{1}, x_{2}, x_{3}, x_{4} \in X$, and $f \in \Phi$.

(i) Working as in the proof of Proposition 3.10(i) we get $\rho \coprod_{f \in \Phi} d_{f}=\coprod_{f \in \Phi} \rho d_{f}$. Since

$$
\begin{aligned}
\left(\rho d_{f}\right)\left(\left[x_{1}\right]_{\sim},\left[x_{2}\right]_{\sim}\right) & =d_{f}\left(x_{1}, x_{2}\right) \\
& =\left|f\left(x_{1}\right)-f\left(x_{2}\right)\right| \\
& =\mid(\rho f)\left(\left[x_{1}\right]_{\sim}\right) \\
& =(\rho f)\left(\left[x_{2}\right]_{\sim}\right) \mid \\
& =d_{\rho f}\left(\left[x_{1}\right]_{\sim},\left[x_{2}\right]_{\sim}\right)
\end{aligned}
$$

we get $\rho d_{f}=d_{\rho f}$, for every $f \in \Phi$, hence $\rho \coprod_{f \in \Phi} d_{f}=\coprod_{f \in \Phi} \rho d_{f}=\coprod_{\rho f \in \rho \Phi} d_{\rho f}$. (ii) We have that

$$
\begin{aligned}
\rho\left(d_{f} \odot \pi_{1}\right)\left(\left(\left[x_{1}\right]_{\sim},\left[x_{2}\right]_{\sim}\right),\left(\left[x_{3}\right]_{\sim},\left[x_{4}\right]_{\sim}\right)\right) & =\rho d_{f \circ \pi_{1}}\left(\left(\left[x_{1}\right]_{\sim},\left[x_{2}\right]_{\sim}\right),\left(\left[x_{3}\right]_{\sim},\left[x_{4}\right]_{\sim}\right)\right) \\
& =d_{f \circ \pi_{1}}\left(\left(x_{1}, x_{2}\right),\left(x_{3}, x_{4}\right)\right) \\
& =\left|f\left(x_{1}\right)-f\left(x_{3}\right)\right| \\
& =d_{f}\left(x_{1}, x_{3}\right) \\
& =\rho d_{f}\left(\left[x_{1}\right]_{\sim},\left[x_{3}\right]_{\sim}\right) \\
& =\left(\rho d_{f} \odot \pi_{1}\right)\left(\left(\left[x_{1}\right]_{\sim},\left[x_{2}\right]_{\sim}\right),\left(\left[x_{3}\right]_{\sim},\left[x_{4}\right]_{\sim}\right)\right)
\end{aligned}
$$


ie $\rho\left(d_{f} \odot \pi_{1}\right)=\rho d_{f} \odot \pi_{1}$. Similarly we get $\rho\left(d_{g} \odot \pi_{2}\right)=\rho d_{g} \odot \pi_{2}$. Using (i) we get

$$
\begin{aligned}
\rho(D \times E) & =\rho\left(\coprod_{f \in \Phi}^{g \in \Theta} d_{f} \odot \pi_{1}, d_{g} \odot \pi_{2}\right) \\
& =\coprod_{f \in \Theta} \rho\left(d_{f} \odot \pi_{1}\right), \rho\left(d_{g} \odot \pi_{2}\right) \\
& =\coprod_{f \in \Theta} \rho d_{f} \odot \pi_{1}, \rho d_{g} \odot \pi_{2} \\
& =\left(\coprod_{f \in \Phi} \rho d_{f}\right) \times\left(\coprod_{g \in \Theta} \rho d_{g}\right) \\
& =\rho D \times \rho E .
\end{aligned}
$$

(iii) If $a_{1}, a_{2} \in A$, then

$$
\begin{aligned}
\rho d_{\left(f_{\mid A}\right)}\left(\left[a_{1}\right]_{\sim},\left[a_{2}\right]_{\sim}\right) & =d_{\left(f_{\mid A}\right)}\left(a_{1}, a_{2}\right) \\
& =\left|f\left(a_{1}\right)-f\left(a_{2}\right)\right| \\
& =d_{f}\left(a_{1}, a_{2}\right) \\
& =\rho d_{f}\left(\left[a_{1}\right]_{\sim},\left[a_{2}\right]_{\sim}\right) \\
& =\left(\rho d_{f}\right)_{\mid A \times A}\left(\left[a_{1}\right]_{\sim},\left[a_{2}\right]_{\sim}\right)
\end{aligned}
$$

ie $\rho d_{\left(f_{\mid A}\right)}=\left(\rho d_{f}\right)_{\mid A \times A}$. Hence by Proposition 3.13(ii) we have that

$$
\rho\left(D_{\mid A}\right)=\rho\left(\coprod_{f \in \Phi} d_{\left(f_{\mid A}\right)}\right)=\coprod_{f \in \Phi} \rho d_{\left(f_{\mid A}\right)}=\coprod_{f \in \Phi}\left(\rho d_{f}\right)_{\mid A \times A}=\left(\coprod_{f \in \Phi} \rho d_{f}\right)_{\mid A}=(\rho D)_{\mid A} .
$$

Theorem 3.15 (Tychonoff embedding theorem for $\mathrm{f}$-uniform spaces) If $\mathcal{D}=$ $\left(X, \coprod_{f \in \Phi} d_{f}\right)$ is an $\mathrm{f}$-uniform space, then $\mathcal{D}$ is separated if and only if $\mathcal{D}$ is uniformly embedded into the Euclidean uniform space $\mathcal{R}^{\Phi}$.

Proof By Proposition 3.13(iii) $\mathcal{D}$ is separated if and only if $\Phi$ is separating. If $\mathcal{D}$ is separated, we define the mapping $\varepsilon_{X}: X \rightarrow \mathbb{R}^{\Phi}$ by

$$
x \mapsto \hat{x}, \quad \hat{x}(f):=f(x)
$$

for every $x \in X$ and $f \in \Phi$. By Proposition 2.21 for a $\Phi$-product we get:

$$
\begin{gathered}
D\left(d_{\mathbb{R}}\right)^{\Phi}=\left(\coprod d_{\mathbb{R}}\right)^{\Phi}=\coprod_{f \in \Phi} d_{\mathbb{R}} \odot \varpi_{f}=\coprod_{f \in \Phi} d_{\varpi_{f}} \\
\left(D\left(d_{\mathbb{R}}\right)^{\Phi}\right)_{\mid \varepsilon_{X}(X)}=\coprod_{f \in \Phi}\left(d_{\varpi_{f}}\right)_{\mid \varepsilon_{X}(X) \times \varepsilon_{X}(X)}
\end{gathered}
$$


If $x, y \in X$ such that $\varepsilon_{X}(x)=\varepsilon_{X}(y) \leftrightarrow \forall_{f \in \Phi}(f(x)=f(y)$, then $x=y$ since $\Phi$ is separating. By the $\amalg$-lifting of morphisms we have that

$$
\varepsilon_{X} \in \operatorname{Mor}\left(\mathcal{D}, \mathcal{R}_{\mid \varepsilon_{X}(X)}^{\Phi}\right) \leftrightarrow \forall_{f \in \Phi}\left(\left(d_{\varpi_{f}}\right)_{\mid \varepsilon_{X}(X) \times \varepsilon_{X}(X)} \odot \varepsilon_{X} \in \coprod_{f \in \Phi} d_{f}\right)
$$

which holds since

$$
\left(d_{\varpi_{f}}\right)_{\mid \varepsilon_{X}(X) \times \varepsilon_{X}(X)} \odot \varepsilon_{X}=d_{\varpi_{f}} \odot \varepsilon_{X}=d_{f}
$$

for every $f \in \Phi$. If $x, y \in X$,

$\left(d_{\varpi_{f}} \odot \varepsilon_{X}\right)(x, y)=d_{\varpi_{f}}(\hat{x}, \hat{y})=\left|\varpi_{f}(\hat{x})-\varpi_{f}(\hat{y})\right|=|\hat{x}(f)-\hat{y}(f)|=|f(x)-f(y)|=d_{f}(x, y)$.

Since $d_{f}=\left(d_{\varpi_{f}}\right)_{\mid \varepsilon_{X}(X) \times \varepsilon_{X}(X)} \odot \varepsilon_{X}$ for every $f \in \Phi$, by the $\amalg$-lifting of openness $\varepsilon_{X}$ is an open morphism from $\mathcal{D}$ to $\left(\mathcal{R}^{\Phi}\right)_{\left.\right|_{\varepsilon_{X}(X)}}$, ie a uniform embedding of $\mathcal{D}$ into $\mathcal{R}^{\Phi}$.

In the previous theorem we avoided the exterior union of sets. If $X=\mathbb{R}^{n}$, then $D\left(d_{\mathbb{R}}\right)^{n}=\coprod d_{\pi_{1}}, \ldots, d_{\pi_{n}}$, ie $\Phi=\left\{\pi_{1}, \ldots, \pi_{n}\right\}$ determines $D\left(d_{\mathbb{R}}\right)^{n}$. If $\vec{x} \in \mathbb{R}^{n}$, for the embedding $\varepsilon_{\mathbb{R}^{n}}$ we have that $\hat{\vec{x}}\left(\pi_{i}\right)=\pi_{i}(\vec{x})=x_{i}$, ie if we identify $\Phi$ with $n$ then $\varepsilon_{\mathbb{R}^{n}}$ is identified with the identity function on $\mathbb{R}^{n}$. Next corollaries are translations of the corresponding results for topological spaces (see Walker [46, pages 6-7]).

Corollary 3.16 If $\mathcal{D}=(X, D)$ is a uniform space, $\mathcal{E}=\left(Y, \coprod_{g \in \Theta} d_{g}\right)$ is a separated $\mathrm{f}$ uniform space and $h \in \operatorname{Mor}(\mathcal{D}, \mathcal{E})$, there exists a mapping $\rho h: \rho X \rightarrow Y \in \operatorname{Mor}(\rho \mathcal{D}, \mathcal{E})$ such that the following diagram commutes:

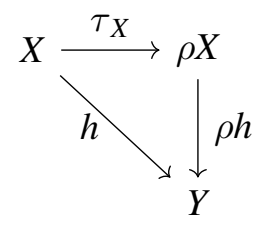

Proof If $e_{Y}$ is the Tychonoff embedding of $\mathcal{E}$ into $\mathcal{R}^{\Theta}$, we define $\mu: \rho X \rightarrow \mathbb{R}^{\Theta}$ by:

$$
\begin{gathered}
{[x]_{\sim} \mapsto \varepsilon_{Y}(h(x))=\widehat{h(x)}} \\
\widehat{h(x)}(g)=g(h(x))
\end{gathered}
$$

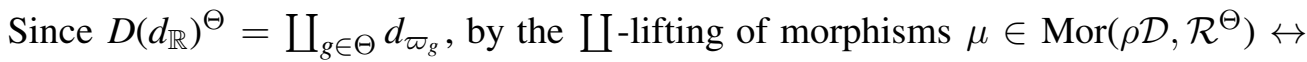
$\forall_{g \in \Theta}\left(d_{\varpi_{g}} \odot \mu \in \rho D\right)$. By Theorem 3.9 we have that

$$
\rho\left(d_{g} \odot h\right) \circ \tau_{X}^{[2]}=d_{g} \circ h^{[2]}
$$


and if $x_{1}, x_{2} \in X$ and $g \in \Theta$, we have that

$$
\begin{aligned}
\left(d_{\varpi_{g}} \odot \mu\right)\left(\left[x_{1}\right]_{\sim},\left[x_{2}\right]_{\sim}\right) & =d_{\varpi_{g}}\left(\widehat{h\left(x_{1}\right)}, \widehat{h\left(x_{2}\right)}\right) \\
& =\left|\varpi_{g}\left(\widehat{h\left(x_{1}\right)}\right)-\varpi_{g}\left(\widehat{h\left(x_{2}\right)}\right)\right| \\
& =\left|\widehat{h\left(x_{1}\right)}(g)-\widehat{h\left(x_{2}\right)}(g)\right| \\
& =\left|g\left(h\left(x_{1}\right)\right)-g\left(h\left(x_{2}\right)\right)\right| \\
& =\left(d_{g} \odot h\right)\left(x_{1}, x_{2}\right) \\
& =\left(\rho\left(d_{g} \odot h\right)\right)\left(\left[x_{1}\right]_{\sim},\left[x_{2}\right]_{\sim}\right)
\end{aligned}
$$

ie $d_{\varpi_{g}} \odot \mu=\rho\left(d_{g} \odot h\right) \in \rho D$. We define $\rho h:=\varepsilon_{Y}^{-1} \circ \mu$, and if $x \in X$ then $\left(\varepsilon_{Y}^{-1} \circ \mu\right)\left([x]_{\sim}\right)=\varepsilon_{Y}^{-1}\left(\varepsilon_{Y}(h(x))=h(x)\right.$.

Corollary 3.17 If $\mathcal{D}=(X, D)$ is a uniform space, $\mathcal{E}=\left(Y, \coprod_{g \in \Theta} d_{g}\right)$ is an $\mathrm{f}$-uniform space, $h \in \operatorname{Mor}(\mathcal{D}, \mathcal{E})$, then there exists a mapping $\rho h: \rho X \rightarrow \rho Y \in \operatorname{Mor}(\rho \mathcal{D}, \rho \mathcal{E})$ such that the following diagram commutes:

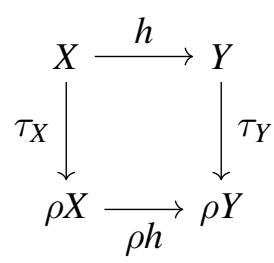

Proof By Proposition 3.14(i) $\rho \mathcal{E}$ is an f-uniform space. Since $\tau_{Y} \circ h \in \operatorname{Mor}(\mathcal{D}, \rho \mathcal{E})$, as a composition of morphisms, by Corollary 3.16 we have that

$$
\rho\left(\tau_{Y} \circ h\right) \circ \tau_{X}=\tau_{Y} \circ h,
$$

therefore $\rho h:=\rho\left(\tau_{Y} \circ h\right)$ is the required mapping.

Proposition 3.18 The full subcategory $\mathrm{f}$-Unif of Unif is reflective in Unif.

Proof If $\mathcal{D}=(X, D)$ is in Unif, we take the $\mathrm{f}$-uniform space $\mathrm{f}-\mathcal{D}=\left(X, \coprod_{h \in \mathcal{M}(\mathcal{D})} d_{h}\right)$. By Proposition 2.27(i) $\coprod_{h \in \mathcal{M}(\mathcal{D})} d_{h} \subseteq D$. The identity function $\operatorname{id}_{X}: X \rightarrow X$ is in $\operatorname{Mor}(\mathcal{D}, f-\mathcal{D})$, since if $h \in \mathcal{M}(\mathcal{D}), d_{h} \odot \mathrm{id}_{X}=d_{h} \in D$. If $\mathcal{E}=\left(Y, \coprod_{f \in \Phi} d_{f}\right)$ is an $\mathrm{f}$-uniform space and $\theta \in \operatorname{Mor}(\mathcal{D}, \mathcal{E})$, then $u=\theta$ is the unique mapping which makes the following diagram to commute: 


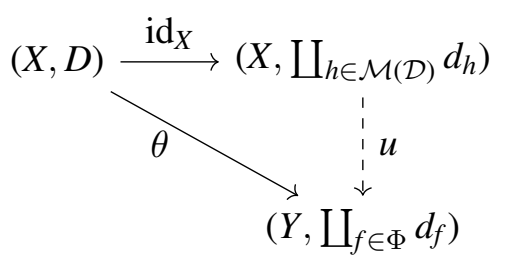

It remains to show (see Mac Lane [27, page 91]) that $u \in \operatorname{Mor}(f-\mathcal{D}, \mathcal{E})$, ie $\forall_{f \in \Phi}\left(d_{f} \odot u \in\right.$ $\left.\coprod_{h \in \mathcal{M}(\mathcal{D})} d_{h}\right)$. If $f \in \Phi$ then $f \circ u=f \circ \theta$, and since $\theta \in \operatorname{Mor}(\mathcal{D}, \mathcal{E}), d_{f} \odot \theta=d_{f \circ \theta}=$ $d_{f \circ u}=d_{f} \odot u \in D$, therefore $f \circ u \in \mathcal{M}(\mathcal{D})$. Consequently, $d_{f} \odot u \in \coprod_{h \in \mathcal{M}(\mathcal{D})} d_{h}$.

One can show similarly that the correspondence $\lambda(X, D)=\left(X, \coprod_{h \in \mathcal{M}(\mathcal{D})} d_{h}\right)$ and $\lambda(\theta)=\theta$ for every $\theta \in \operatorname{Mor}(\mathcal{D}, \mathcal{E})$, is a covariant functor from Unif to $f$-Unif .

Theorem 3.19 If $\mathcal{D}=(X, D)$ is a separated uniform space, $\mathcal{E}=(Y, E)$ is a uniform space, and $\tau \in \operatorname{Mor}(\mathcal{D}, \mathcal{E})$, the following are equivalent:

(i) $\tau$ is open.

(ii) The induced mapping $T: E \rightarrow D$ of $\tau$ is onto $D$.

(iii) $\tau$ is a uniform embedding of $\mathcal{D}$ into $\mathcal{E}$ such that

$$
\mathcal{E}_{\tau(X)}=\left\{e_{\mid \tau(X) \times \tau(X)} \mid e \in E\right\} .
$$

Proof The equivalence between (i) and (ii) is immediate. We suppose that $\tau$ is open, and we show first that $\tau$ is an injection. If $x_{1}, x_{2} \in X$ such that $\tau\left(x_{1}\right)=\tau\left(x_{2}\right)$, we show that $\forall_{d \in D}\left(d\left(\left(x_{1}, x_{2}\right)=0\right)\right.$, so that, since $D$ is separating, $x_{1}=x_{2}$. If $d \in D$, by hypothesis there exists $e \in E$ such that $d=e \odot \tau$, hence $d\left(x_{1}, x_{2}\right)=(e \odot \tau)\left(x_{1}, x_{2}\right)=$ $e\left(\tau\left(x_{1}\right), \tau\left(x_{2}\right)\right)=0$. Since $\tau$ is open as a morphism from $\mathcal{D}$ to $\mathcal{E}$, by Proposition 2.23(ii) it is open as a morphism from $\mathcal{D}$ onto $\mathcal{E}_{\mid \tau(X)}$, ie it is a uniform embedding from $\mathcal{D}$ into $\mathcal{E}$. Clearly, $\left\{e_{\mid \tau(X) \times \tau(X)} \mid e \in E\right\} \subseteq \mathcal{E}_{\tau(X)}$. The inclusion

$$
\coprod_{e \in E} e_{\mid \tau(X) \times \tau(X)} \subseteq\left\{e_{\mid \tau(X) \times \tau(X)} \mid e \in E\right\}
$$

follows immediately by showing that $\left\{e_{\mid \tau(X) \times \tau(X)} \mid e \in E\right\}$ is a uniformity. Clearly, $\overline{0}=\overline{0}_{\mid \tau(X) \times \tau(X)}$. If $e_{1}, e_{2} \in E$, then

$$
e_{1 \mid \tau(X) \times \tau(X)} \vee e_{2 \mid \tau(X) \times \tau(X)}=\left(e_{1} \vee e_{2}\right)_{\mid \tau(X) \times \tau(X)} .
$$

If $e \in E$ and $e^{\prime} \in \mathbb{D}(\tau(X))$ such that $U\left(e_{\mid \tau(X) \times \tau(X)}, \delta, e^{\prime}, \epsilon\right)$, then $U\left(e \odot \tau, \delta, e^{\prime} \odot \tau, \epsilon\right)$. Since this is the case for every $\epsilon>0$, we get $e^{\prime} \odot \tau \in D$. By hypothesis there exists 
$e^{\prime \prime} \in E$ such that $e^{\prime} \odot \tau=e^{\prime \prime} \odot \tau=e^{\prime \prime}{ }_{\mid \tau(X) \times \tau(X)} \odot \tau$, hence by Proposition 2.14(viii) $e^{\prime}=e^{\prime \prime}{ }_{\mid \tau(X) \times \tau(X)}$, ie $e^{\prime} \in\left\{e_{\mid \tau(X) \times \tau(X)} \mid e \in E\right\}$. For the converse implication, since $\tau$ is an isomorphism between $\mathcal{D}$ and $\mathcal{E}_{\tau(X)}$, if $d \in D$ there exists $e^{\prime} \in\left\{e_{\mid \tau(X) \times \tau(X)} \mid e \in E\right\}$ such that $d=e^{\prime} \odot \tau$, ie there exists $e \in E$ such that $d=e_{\mid \tau(X) \times \tau(X)} \odot \tau=e \odot \tau$, so $\tau$ is open as a morphism from $\mathcal{D}$ to $\mathcal{E}$.

\section{From Bishop spaces to uniform spaces}

In this section we study the relationship between a Bishop space and its generated uniform space. First we give a definition that corresponds to Definition 2.3 using the letter $U$ for both relations $U(d, \delta, e, \epsilon)$ and $U(g, f, \epsilon)$ to stress the similarity in the development of the theories of uniformities of pseudometrics and of Bishop topologies.

Definition 4.1 If $f, g \in \mathbb{F}(X), \Phi \subseteq \mathbb{F}(X)$, and $\epsilon>0$, the uniform closure $\bar{\Phi}$ of $\Phi$ is:

$$
\begin{gathered}
\bar{\Phi}:=\{f \in \mathbb{F}(X) \mid U(\Phi, f)\} \\
U(\Phi, f):=\forall_{\epsilon>0} \exists_{g \in \Phi}(U(g, f, \epsilon)) \\
U(g, f, \epsilon):=\forall_{x \in X}(|f(x)-g(x)| \leq \epsilon)
\end{gathered}
$$

We denote by $\mathrm{B}(\mathbb{R})$ the set of all Bishop continuous functions of type $\mathbb{R} \rightarrow \mathbb{R}$, ie those which are uniformly continuous on every bounded subset $B$ of $\mathbb{R}$, with a modulus of continuity $\omega_{\phi, B}(\epsilon)$ for every $\epsilon>0$, ie

$$
\forall_{x, y \in B}\left(|x-y| \leq \omega_{\phi, B}(\epsilon) \rightarrow|\phi(x)-\phi(y)| \leq \epsilon\right) .
$$

Definition 4.2 A Bishop space is a pair $(X, F)$, where $F \subseteq \mathbb{F}(X)$ is a Bishop topology of functions on $X$ satisfying the following conditions:

$$
\begin{array}{ll}
\left(\mathrm{BS}_{1}\right) & a \in \mathbb{R} \rightarrow \bar{a}_{X} \in F . \\
\left(\mathrm{BS}_{2}\right) & f, g \in F \rightarrow f+g \in F . \\
\left(\mathrm{BS}_{3}\right) & f \in F \rightarrow \phi \in \mathrm{B}(\mathbb{R}) \rightarrow \phi \circ f \in F . \\
\left(\mathrm{BS}_{4}\right) & \bar{F}=F .
\end{array}
$$

If $\mathcal{G}=(Y, G)$ is a Bishop space, a function $h: X \rightarrow Y$ is a Bishop morphism if $\forall_{g \in G}(g \circ h \in F)$ :

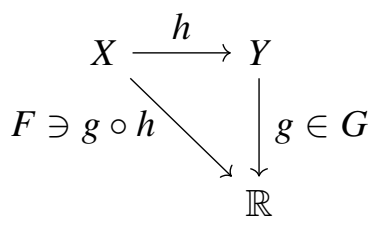


We denote by $\operatorname{Mor}(\mathcal{F}, \mathcal{G})$ the set of all Bishop morphisms from $\mathcal{F}$ to $\mathcal{G}$. The Bishop morphisms are the arrows in the category of Bishop spaces Bis. The Bishop space

$$
\mathcal{R}:=(\mathbb{R}, \mathrm{B}(\mathbb{R}))
$$

is called the Bishop space of reals, ${ }^{12}$ and $\mathrm{B}^{*}(\mathbb{R})$ denotes the set of bounded elements of $\mathrm{B}(\mathbb{R})$. We use the notations $\mathcal{M}(\mathcal{F})$ for $\operatorname{Mor}(\mathcal{F}, \mathcal{R})$ and $\mathcal{M}^{*}(\mathcal{F})$ for the bounded elements of $\mathcal{M}(\mathcal{F})$. A topology $F$ is called pseudo-compact, if every element of $F$ is a bounded function. We denote by $F^{*}$ the topology of bounded elements of $F$.

It is immediate to see that $\operatorname{Const}(X)$ and $\mathbb{F}(X)$ are topologies on $X$, which we call the trivial and the discrete topology on $X$, respectively, and that if $F$ is a topology on $X$ then $\operatorname{Const}(X) \subseteq F \subseteq \mathbb{F}(X)$. Moreover, if $F$ is a topology on $X, F^{*}=F \cap \mathcal{F}^{*}(X)$ is a topology on $X$. A Bishop topology $F$ is a ring and a lattice; since $\left|\operatorname{id}_{\mathbb{R}}\right| \in \mathrm{B}(\mathbb{R})$, where $\mathrm{id}_{\mathbb{R}}$ is the identity function on $\mathbb{R}$, by $\mathrm{BS}_{3}$ we get that if $f \in F$ then $|f| \in F$. By $\mathrm{BS}_{2}$ and $\mathrm{BS}_{3}$ we also get that if $f, g \in F$, then $f \cdot g, f \vee g, f \wedge g \in F$ (see Bishop and Bridges [7, page 77]).

Definition 4.3 The Bishop closure of $F_{0}$, or the least topology $\bigvee F_{0}$ generated by some $F_{0} \subseteq \mathbb{F}(X)$, is defined by the following inductive rules:

$$
\begin{gathered}
\frac{f_{0} \in F_{0}}{f_{0} \in \bigvee F_{0}} \quad \frac{a \in \mathbb{R}}{\bar{a}_{X} \in \bigvee F_{0}} \quad \frac{f, g \in \bigvee F_{0}}{f+g \in \bigvee F_{0}} \\
\frac{f \in \bigvee F_{0}, \phi \in \mathrm{B}(\mathbb{R})}{\phi \circ f \in \bigvee F_{0}} \quad \frac{\left(g \in \bigvee F_{0}, U(g, f, \epsilon)\right)_{\epsilon>0}}{f \in \bigvee F_{0}}
\end{gathered}
$$

We call $F_{0}$ a subbase of $\bigvee F_{0}$, and we also call $\bigvee F_{0}$ the Bishop closure of $F_{0}$.

Note that if $F_{0}$ is inhabited, then the rule of the inclusion of the constant functions is redundant to the rule of closure under composition with $\mathrm{B}(\mathbb{R})$. The most complex inductive rule above can be replaced by the rule:

$$
\frac{g_{1} \in \bigvee F_{0} \wedge U\left(g_{1}, f, \frac{1}{2}\right), g_{2} \in \bigvee F_{0} \wedge U\left(g_{2}, f, \frac{1}{2^{2}}\right), \ldots}{f \in \bigvee F_{0}}
$$

\footnotetext{
${ }^{12}$ We use for simplicity the same symbol $\mathcal{R}$ for the uniform space of reals and for the Bishop space for reals, as the meaning of the symbol is going to be clear in every context. What corresponds to Proposition 2.28(i) is that if $F$ is a topology on $X$, then $F=\operatorname{Mor}(\mathcal{F}, \mathcal{R})$.
} 
The above rules induce the following induction principle $\operatorname{Ind}_{\mathcal{F}}$ on $\bigvee F_{0}$ :

$$
\begin{aligned}
& \forall_{f_{0} \in F_{0}}\left(P\left(f_{0}\right)\right) \rightarrow \\
& \forall_{a \in \mathbb{R}}\left(P\left(\bar{a}_{X}\right)\right) \rightarrow \\
& \forall_{f, g \in \bigvee F_{0}}(P(f) \rightarrow P(g) \rightarrow P(f+g)) \rightarrow \\
& \forall_{f \in \bigvee F_{0}} \forall_{\phi \in \mathrm{B}(\mathbb{R})}(P(f) \rightarrow P(\phi \circ f)) \rightarrow \\
& \forall_{f \in \bigvee F_{0}}\left(\forall_{\epsilon>0} \exists_{g \in \bigvee F_{0}}(P(g) \wedge U(g, f, \epsilon)) \rightarrow P(f)\right) \rightarrow \\
& \forall_{f \in \bigvee F_{0}}(P(f))
\end{aligned}
$$

where $P$ is any property on $\mathbb{F}(X)$. Through $\operatorname{Ind}_{\mathcal{F}}$ one shows the $\bigvee$-lifting of Bishop morphisms: a function $h: X \rightarrow Y \in \operatorname{Mor}\left(\mathcal{F}, \mathcal{G}_{0}\right)$ if and only if $\forall_{g_{0} \in G_{0}}\left(g_{0} \circ h \in F\right)$ :

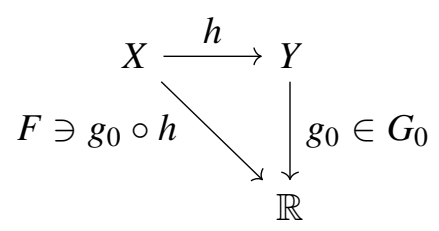

Definition 4.4 If $F$ is a topology on $X$ an $F_{0} \subseteq F$ such that $F=\bigvee F_{0}$ is called a subbase for $F$. A $\Phi \subseteq F$ such that $\bar{\Phi}=F$ is called a base for $F$. A topology $F$ is called tight if the canonical point-point apartness relation induced by $F$, defined by $x \bowtie_{F} y: \leftrightarrow \forall_{f \in F}(f(x)=f(y))$ for every $x, y \in X$, is tight. A Bishop space with a tight topology is called a separated Bishop space.

As expected, a topology $F$ is tight if and only if $F$ it is separating (Definition 3.11).

Definition 4.5 Let $\mathcal{F}=(X, F), \mathcal{G}=(Y, G)$ be Bishop spaces, $A \subseteq X$ be inhabited, and $\phi: X \rightarrow Y$ be onto $Y$. The product Bishop space $\mathcal{F} \times \mathcal{G}=(X \times Y, F \times G)$ of $\mathcal{F}$ and $\mathcal{G}$, relative Bishop space $\mathcal{F}_{\mid A}=\left(A, F_{\mid A}\right)$ on $A$, and the quotient topology $G_{\phi}$ on $Y$ are defined, respectively, by:

$$
\begin{gathered}
F \times G:=\bigvee\left[\left\{f \circ \pi_{1}, \mid f \in F\right\} \cup\left\{g \circ \pi_{2} \mid g \in G\right\}\right]=: \bigvee_{f \in F}^{g \in G} f \circ \pi_{1}, g \circ \pi_{2} \\
F_{\mid A}:=\bigvee\left\{f_{\mid A} \mid f \in F\right\}=: \bigvee_{f \in F} f_{\mid A} \\
F_{\phi}:=\{g \in \mathbb{F}(Y) \mid g \circ \phi \in F\}
\end{gathered}
$$

Journal of Logic \& Analysis 11:FT2 (2019) 
As in the case of uniform spaces one shows inductively that

$$
\begin{aligned}
\bigvee F_{0} \times \bigvee G_{0} & :=\bigvee\left[\left\{f_{0} \circ \pi_{1}, \mid f_{0} \in F_{0}\right\} \cup\left\{g_{0} \circ \pi_{2} \mid g_{0} \in G_{0}\right\}\right] \\
& =: \bigvee_{f_{0} \in F_{0}} f_{0} \circ \pi_{1}, g_{0} \circ \pi_{2}, \\
\left(\bigvee F_{0}\right)_{\mid A} & =\bigvee\left\{f_{0 \mid A} \mid f_{0} \in F_{0}\right\}=: \bigvee_{f_{0} \in F_{0}} f_{0 \mid A} .
\end{aligned}
$$

and

The next proposition, the proof of which is omitted as straightforward, shows the relation between the elements of $\mathbb{F}(X)$ and their induced pseudometrics.

Proposition 4.6 Suppose that $f, g \in \mathbb{F}(X), a, c>0, b \in \mathbb{R}$, and $\phi \in \mathrm{B}(\mathbb{R})$.

(i) $d_{\bar{a}_{X}}=\overline{0}_{X \times X}$.

(ii) $d_{f+g} \leq d_{f}+d_{g}$.

(iii) $d_{f+\bar{b}_{X}}=d_{f}$.

(iv) $d_{\bar{a}_{X} h}=\bar{a}_{X} d_{h}$.

(v) $U\left(g, f, \frac{\epsilon}{3}\right) \rightarrow U\left(d_{g}, \frac{\epsilon}{3}, d_{f}, \epsilon\right)$.

(vi) If $f$ is bounded, then $U\left(d_{f}, \omega_{\phi, f(X)}(\epsilon), d_{\phi \circ f}, \epsilon\right)$.

(vii) If $|f| \geq \bar{c}_{X}$, then $d_{\frac{1}{f}} \leq \frac{1}{c^{2}} d_{f}$.

The next proposition describes the "canonical" uniform space of pseudometrics generated by some Bishop space and it has a categorical formulation.

Proposition 4.7 Let $\mathcal{F}=(X, F)$ and $\mathcal{G}=(Y, G)$ be Bishop spaces. The uniform space generated by $\mathcal{F}$ is the pair $\mathcal{D}(\mathcal{F})=(X, D(F))$, where:

$$
D(F):=\coprod\left\{d_{f} \mid f \in F\right\}=: \coprod_{f \in F} d_{f}
$$

(i) The mapping $\tau$ which sends $\mathcal{F}$ to $\tau(\mathcal{F})=\mathcal{D}(\mathcal{F})$ and a function $h \in \operatorname{Mor}(\mathcal{F}, \mathcal{G})$ to $\tau(h)=h \in \operatorname{Mor}(\tau(\mathcal{F}), \tau(\mathcal{G}))$ is a covariant functor from Bis to $\mathbf{f}$-Unif .

(ii) $D(\operatorname{Const}(X))=\left\{\overline{0}_{X \times X}\right\}$, and $D(\mathbb{F}(X))=\coprod_{f \in \mathbb{F}(X)} d_{f}$.

(iii) $F$ is separating if and only if $D(F)$ is separating.

Proof (i) If $h \in \operatorname{Mor}(\mathcal{F}, \mathcal{G})$, then $h \in \operatorname{Mor}(\tau(\mathcal{F}), \tau(\mathcal{G})) \leftrightarrow \forall_{g \in G}\left(d_{g} \odot h \in D(F)\right)$. If $g \in G$, since $d_{g}=d_{\mathbb{R}} \odot g$, by Proposition 2.14(vii) we have that $d_{g} \odot h=\left(d_{\mathbb{R}} \odot g\right) \odot g=$ $d_{\mathbb{R}} \odot(g \circ h)=d_{g \circ h}$. Since $h \in \operatorname{Mor}(\mathcal{F}, \mathcal{G}), g \circ h \in F$, hence $d_{g \circ h} \in D(F)$. It is immediate 
that $\tau\left(\operatorname{id}_{X}\right)=\operatorname{id}_{\tau(X)}$ and if $h \in \operatorname{Mor}(\mathcal{F}, \mathcal{G}), h^{\prime} \in \operatorname{Mor}(\mathcal{G}, \mathcal{H})$, where $\mathcal{H}=(Z, H)$ is a Bishop space, then $\tau\left(h^{\prime} \circ h\right)=\tau\left(h^{\prime}\right) \circ \tau(h)$.

(ii) This is immediate by Proposition 4.6(i) and by the definition of $D(F)$.

(iii) By Proposition $3.3 D(F)$ is separating if and only if $\forall_{x, y \in X}\left(\forall_{f \in F}\left(d_{f}(x, y)=0\right) \rightarrow\right.$ $x=y) \leftrightarrow \forall_{x, y \in X}\left(\forall_{f \in F}(f(x)=f(y)) \rightarrow x=y\right)$, the separating property of $F$.

Proposition 4.8 If $F_{0}$ is a subbase of a pseudo-compact topology $F$ on $X$, then:

$$
D\left(\bigvee F_{0}\right)=\coprod_{f_{0} \in F_{0}} d_{f_{0}}
$$

Proof It suffices to show that $\coprod_{f \in F} d_{f} \subseteq \coprod_{f_{0} \in F_{0}} d_{f_{0}}$. This we show using induction on $\bigvee F_{0}$. For the cases $f=\bar{a}_{X}$, where $a \in \mathbb{R}, f=f_{1}+f_{2}$, and $U\left(g, f, \frac{\epsilon}{3}\right)$ we use the inductive hypotheses, the basic properties of a uniformity and Proposition 4.6(i), (ii), and (iii), respectively. If $f=\phi \circ g$, where $\phi \in \mathrm{B}(\mathbb{R})$ and $g \in \bigvee F_{0}$ such that $d_{g} \in \coprod_{f_{0} \in F_{0}} d_{f_{0}}$, then by Proposition 4.6(iv) we get $U\left(d_{g}, \omega_{\phi, g(X)}(\epsilon), d_{\phi \circ g}, \epsilon\right)$, and since $\epsilon>0$ is arbitrary we conclude that $d_{\phi \circ g} \in \coprod_{f_{0} \in F_{0}} d_{f_{0}}$.

Corollary 4.9 (i) If $\tau$ is restricted to the full subcategory of pseudo-compact Bishop spaces Bis* of Bis, then $\tau$ preserves products and subspaces.

(ii) If $\mathcal{F}=(X, F)$ is a Bishop space and $\mathcal{F}_{\phi}=\left(Y, F_{\phi}\right)$ is the quotient Bishop space with respect to the surjection $\phi: X \rightarrow Y$, then $D\left(F_{\phi}\right) \subseteq D(F)_{\phi}$.

Proof (i) Let $\mathcal{F}=(X, F), \mathcal{G}=(Y, G)$ be in Bis* and $A \subseteq X$ inhabited. Since boundedness of functions is a lifted property from a subbase for a Bishop topology to the topology itself, $\mathcal{F} \times \mathcal{G}$ and $\mathcal{F}_{\mid A}$ are in Bis*. Since $d_{f} \odot \pi_{1}=d_{f \circ \pi_{1}}$ and $d_{g} \odot \pi_{2}=d_{g \circ \pi_{2}}$ :

$$
\begin{aligned}
D(F \times G) & =\coprod_{f \in F}^{g \in G} d_{f \circ \pi_{1}}, d_{g \circ \pi_{2}} \\
& =\coprod_{f \in F}^{g \in G} d_{f} \odot \pi_{1}, d_{g} \odot \pi_{2} \\
& =\left(\coprod_{f \in F} d_{f} \odot \pi_{1}\right) \times\left(\coprod_{g \in G} d_{g} \odot \pi_{2}\right) \\
& =D(F) \times D(G)
\end{aligned}
$$

Since $d_{\left(f_{\mid A}\right)}=\left(d_{f}\right)_{\mid(A \times A)}$, we have that:

$$
D\left(F_{\mid A}\right)=\coprod_{f \in F} d_{\left(f_{\mid A}\right)}=\coprod_{f \in F}\left(d_{f}\right)_{\mid(A \times A)}=D(F)_{\mid A}
$$


(ii) By definition:

$$
\begin{aligned}
& D\left(F_{\phi}\right)=\coprod_{g \in F_{\phi}} d_{g}=\coprod_{g \in \mathbb{F}(Y), g \circ \phi \in F} d_{g} \\
& D(F)_{\phi}=\{e \in \mathbb{D}(Y) \mid e \odot \phi \in D(F)\}
\end{aligned}
$$

If $g \in \mathbb{F}(Y)$ with $g \circ \phi \in F$, then $d_{g} \odot \phi=d_{g \circ \phi} \in \coprod_{f \in F} d_{f}=D(F)$.

Corollary 4.10 Let $\mathcal{F}=(X, F)$ be a separated Bishop space.

(i) The uniform space $\tau(\mathcal{F})$ is embedded into the Euclidean uniform space $\mathcal{R}^{F}$.

(ii) If $F_{0}$ is a subbase for $F, \tau(\mathcal{F})$ is embedded into the Euclidean uniform space $\mathcal{R}^{F_{0}}$.

Proof (i) By Proposition 4.7(iii) $\tau(\mathcal{F})$ is separated, and we use Theorem 3.15.

(ii) By Proposition 4.8 $D(F)=\coprod_{f_{0} \in F_{0}} d_{f_{0}}$. It is easy to show that $F$ is separating if and only if $F_{0}$ is separating, and then we use Theorem 3.15.

Proposition 4.11 Let $(X, F)$ be a Bishop space, $f, f^{\prime} \in F$, and $h \in \mathbb{F}(X)$ a positively non-constant function, ie $h\left(x_{0}\right) \bowtie_{\mathbb{R}} h\left(y_{0}\right) \leftrightarrow\left|h\left(x_{0}\right)-h\left(y_{0}\right)\right|>0$ for some $x_{0}, y_{0} \in X$.

(i) If $d_{h}=d_{f}$, then $h \in F$.

(ii) If $d_{h}=d_{f} \vee d_{f^{\prime}}$, then $h \in F$.

Proof Let $g:=h-{\overline{h\left(x_{0}\right)}}_{X}$.

(i) Since $|g|=\left|h-{\overline{h\left(x_{0}\right)_{X}}}\right|=\left|f-{\overline{f\left(x_{0}\right)_{X}}}\right| \in F,|g|^{2}=g^{2} \in F$. Moreover,

$$
\begin{aligned}
g-\overline{g\left(y_{0}\right)_{X}} & =\left(h-{\overline{h\left(x_{0}\right)_{X}}}\right)-\overline{h\left(y_{0}\right)-h\left(x_{0}\right)_{X}} \\
& =h-{\overline{h\left(x_{0}\right)_{X}}}-{\overline{h\left(y_{0}\right)_{X}}}+{\overline{h\left(x_{0}\right)_{X}}}=h-{\overline{h\left(y_{0}\right)_{X}}} .
\end{aligned}
$$

Hence:

$$
\begin{aligned}
\left|g-{\overline{g\left(y_{0}\right)}}_{X}\right| & =\left|h-{\overline{h\left(y_{0}\right)_{X}}}\right|=\left|f-{\overline{f\left(y_{0}\right)_{X}}}\right| \in F \\
\left|g-{\overline{g\left(y_{0}\right)}}_{X}\right|^{2} & =\left(g-{\overline{g\left(y_{0}\right)_{X}}}^{2}\right)^{2}=g^{2}-2 g{\overline{g\left(y_{0}\right)_{X}}}+{\overline{g\left(y_{0}\right)_{X}}}_{X} \in F
\end{aligned}
$$

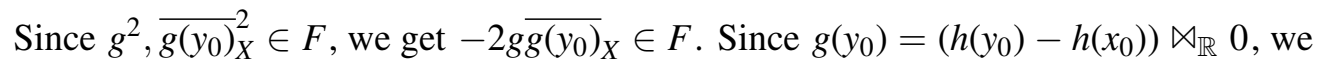
get $g \in F$, hence $h \in F$.

(ii) Since $|g|=\left|h-{\overline{h\left(x_{0}\right)}}_{X}\right|=\left|f-{\overline{f\left(x_{0}\right)_{X}}}\right| \vee\left|g-{\overline{g\left(x_{0}\right)}}_{X}\right| \in F$, we work as in (i). 
As in the case of uniform spaces one can show that any Bishop topology $F$ on some $X$ is isomorphic as an algebra and a lattice to a separating topology $\rho F$ on $\rho X$. If we define the equivalence relation $x_{1} \approx x_{2} \leftrightarrow \forall_{f \in F}\left(f\left(x_{1}\right)=f\left(x_{2}\right)\right)$, for every $x_{1}, x_{2} \in X$ (Definition 3.11), and if $\tau=\pi: X \rightarrow X / \approx$, where $x \mapsto[x] \approx$, then if $\rho X=X / \approx$ is endowed with the quotient Bishop topology $\rho F=\{\rho f \mid f \in F\}=G_{\pi}$, where $(\rho f)\left([x]_{\approx}\right)=f(x)$, for every $[x]_{\approx} \in \rho X$, the following theorem is proved (see also [32]).

Theorem 4.12 (Stone-Čech theorem for Bishop spaces) If $\mathcal{F}=(X, F)$ is a Bishop space, there is a Bishop space $\rho \mathcal{F}=(\rho X, \rho F)$ and a mapping $\tau_{X}: X \rightarrow \rho X \in$ $\operatorname{Mor}(\mathcal{F}, \rho \mathcal{F})$ such that the following hold.

(i) The topology $\rho F$ is separating.

(ii) The induced mapping $T_{X}: \rho F \rightarrow F$ of $\tau_{X}$ is an algebra and lattice isomorphism.

(iii) If $\in F$, there is a unique $\rho f \in \rho F$ such that the following diagram commutes:

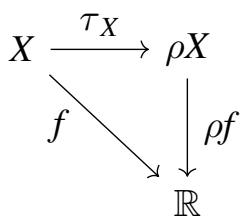

Proposition 4.13 If $\mathcal{F}=(X, F)$ is a Bishop space, then $\rho \mathcal{D}(\mathcal{F})=\mathcal{D}(\rho \mathcal{F})$.

Proof By definition $\rho \mathcal{D}(\mathcal{F})=(\rho X, \rho D(F))$ and $\mathcal{D}(\rho \mathcal{F})=(\rho X, D(\rho F))$, where since $\forall_{f \in F}\left(f\left(x_{1}\right)=f\left(x_{2}\right)\right) \leftrightarrow \forall_{f \in F}\left(d_{f}\left(x_{1}, x_{2}\right)=0\right)$, for every $x_{1}, x_{2} \in X$ the carrier sets $\rho X$ in both structures are the same and therefore the same notation is justified. Moreover, $\left(\rho d_{f}\right)\left(\left[x_{1}\right]_{\approx},\left[x_{2}\right]_{\approx}\right)=d_{f}\left(x_{1}, x_{2}\right)=\left|f\left(x_{1}\right)-f\left(x_{2}\right)\right|=\left|(\rho f)\left(\left[x_{1}\right]_{\approx}\right)=(\rho f)\left(\left[x_{2}\right] \approx\right)\right|=$ $d_{\rho f}\left(\left[x_{1}\right]_{\approx},\left[x_{2}\right] \approx\right)$, eg $\rho d_{f}=d_{\rho f}$ for every $f \in F$. By Proposition 3.14(i) we get

$$
\rho D(F)=\rho\left(\coprod_{f \in F} d_{f}\right)=\coprod_{f \in F} \rho d_{f}=\coprod_{f \in F} d_{\rho f}=D(\{\rho f \mid f \in F\})=D(\rho F) .
$$

Defining the notion of topological embedding of a Bishop spaces into another, and the notion of a Euclidean Bishop space $\mathcal{R}^{I}$ in the obvious way, the same embedding $e_{X}: X \rightarrow \mathbb{R}^{F}$ together with the corresponding $\bigvee$-lifting of openness for Bishop morphisms show the Tychonoff embedding theorem for Bishop spaces (see [32]).

Theorem 4.14 (Tychonoff embedding theorem for Bishop spaces) If $\mathcal{F}=(X, F)$ is a Bishop space, $F$ is separating if and only if $\mathcal{F}$ is topologically embedded into the Euclidean Bishop space $\mathcal{R}^{F}$. 
Proposition 4.15 If $\mathcal{F}=(X, F)$ is a Bishop space, the following diagram commutes:

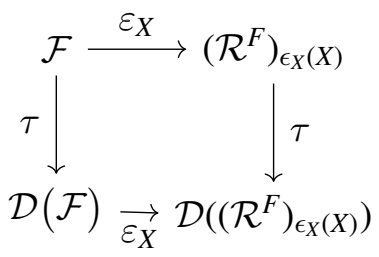

Proof By definition and by our remark after Definition 4.5 we have that

$$
\left(\mathrm{B}(\mathbb{R})^{F}\right)_{\varepsilon_{X}(X)}=\bigvee_{f \in F}\left(\varpi_{f}\right)_{\mid \varepsilon_{X}(X)},
$$

while by Proposition 4.8 we have that

$$
D\left(\left(\mathrm{~B}(\mathbb{R})^{F}\right)_{\varepsilon_{X}(X)}\right)=\coprod_{f \in F} d_{\left[\left(\varpi_{f}\right)_{\mid \varepsilon_{X}(X)}\right]} .
$$

Since $d_{\left[\left(\varpi_{f}\right)_{\mid \varepsilon_{X}(X)}\right.}=\left(d_{\varpi_{f}}\right)_{\mid \varepsilon_{X}(X) \times \varepsilon_{X}(X)}$, we get that $D\left(\left(\mathrm{~B}(\mathbb{R})^{F}\right)_{\varepsilon_{X}(X)}\right)=\left(\mathcal{R}^{F}\right)_{\mid \varepsilon_{X}(X)}$.

\section{From uniform spaces to Bishop spaces}

In this section we study a pseudo-compact Bishop topology generated by a uniformity.

Proposition 5.1 Let $\mathcal{D}=(X, D)$ be a uniform space.

(i) $a \in \mathbb{R} \rightarrow \bar{a}_{X} \in \mathcal{M}(\mathcal{D})$.

(ii) $h_{1}, h_{2} \in \mathcal{M}(\mathcal{D}) \rightarrow h_{1}+h_{2} \in \mathcal{M}(\mathcal{D})$.

(iii) $\overline{\mathcal{M}(\mathcal{D})}=\mathcal{M}(\mathcal{D})$, where $\overline{\mathcal{M}(\mathcal{D})}$ is the uniform closure of $\mathcal{M}(\mathcal{D})$.

(iv) If $h \in \mathcal{M}(\mathcal{D})$ and $a>0$, then $\bar{a}_{X} h \in \mathcal{M}(\mathcal{D})$.

(v) If $h \in \mathcal{M}(\mathcal{D})$ and $c>0$ such that $|h| \geq \bar{c}_{X}$, then $\frac{1}{h} \in \mathcal{M}(\mathcal{D})$.

(vi) If $h \in \mathcal{M}^{*}(\mathcal{D}) \rightarrow \phi \in \mathrm{B}(\mathbb{R}) \rightarrow \phi \circ h \in \mathcal{M}^{*}(\mathcal{D})$.

(vii) $\mathcal{M}^{*}(\mathcal{D})$ is a pseudo-compact Bishop topology on $X$.

Proof (i)-(vi) follow immediately by Proposition 4.6, by Proposition 2.27(i) and by Proposition 2.7. Case (vii) follows immediately from (i)-(vi). 
Proposition 5.2 Let $\mathcal{D}=(X, D)$ and $\mathcal{E}=(Y, E)$ be uniform spaces. The pseudocompact Bishop space generated by $\mathcal{D}$ is the pair $\mathcal{F}(\mathcal{D})=(X, F(D))$, where

$$
F(D):=\mathcal{M}^{*}(\mathcal{D}) \text {. }
$$

(i) The mapping $\nu$ which sends $\mathcal{D}$ to $\nu(\mathcal{D})=\mathcal{F}(\mathcal{D})$ and a function $h \in \operatorname{Mor}(\mathcal{D}, \mathcal{E})$ to $\nu(h)=h \in \operatorname{Mor}(\nu(\mathcal{D}), \nu(\mathcal{E}))$ is a covariant functor from Unif to Bis*.

(ii) $F\left(\left\{\overline{0}_{X \times X}\right\}\right)=\operatorname{Const}(X)$, and $F(\mathbb{D}(X))=\mathbb{F}^{*}(X)$.

(iii) $D$ is separating if and only if $F(D)$ is separating.

Proof (i) If $h \in \operatorname{Mor}(\mathcal{D}, \mathcal{E})$, ie if $\forall_{e \in E}(e \odot h \in D)$, then

$$
\begin{aligned}
h \in \operatorname{Mor}(\nu(\mathcal{D}), \nu(\mathcal{E})) & \leftrightarrow \forall_{\left.g \in \mathcal{M}^{*}(\mathcal{E})\right)}\left(g \circ h \in \mathcal{M}^{*}(\mathcal{D})\right) \\
& \leftrightarrow \forall_{g \in \mathbb{F}^{*}(Y)}\left(d_{g} \in E \rightarrow d_{g \circ h} \in D\right)
\end{aligned}
$$

which is the case, since $d_{g \circ h}=d_{g} \odot h$. It is immediate that $\nu\left(\operatorname{id}_{X}\right)=\mathrm{id}_{\nu(X)}$, and if $h \in \operatorname{Mor}(\mathcal{D}, \mathcal{E}), h^{\prime} \in \operatorname{Mor}(\mathcal{E}, \mathcal{B})$, where $\mathcal{B}=(Z, B)$ is a uniform space, then $\nu\left(h^{\prime} \circ h\right)=\nu\left(h^{\prime}\right) \circ \nu(h)$.

(ii) Since $h \in \mathcal{M}^{*}\left(X,\left\{\overline{0}_{X \times X}\right\}\right) \leftrightarrow d_{h}=\overline{0}_{X \times X}$, we show $d_{h}=\overline{0}_{X \times X} \leftrightarrow h \in \operatorname{Const}(X)$. The less trivial implication is $d_{h}=\overline{0}_{X \times X} \rightarrow h \in \operatorname{Const}(X)$. If $x_{0}$ inhabits $X$, and if $h\left(x_{0}\right)=a$, then $h=\bar{a}_{X}$, since if $x \in X$ then $d_{h}\left(x, x_{0}\right)=0 \leftrightarrow h(x)=h\left(x_{0}\right)=a$. For the second equality we have that a bounded function $h: X \rightarrow \mathbb{R}$ is in $\mathcal{M}^{*}(X, \mathbb{D}(X))$ if and only if $d_{h} \in \mathbb{D}(X)$ if and only if $h \in \mathbb{F}^{*}(X)$.

(iii) Let $x, y \in X$. Suppose that $D$ is separating and $\forall_{h \in \mathcal{M}^{*}(\mathcal{D})}(h(x)=h(y))$. It suffices to show $\forall_{d \in D}(d(x, y)=0)$. If $d \in D$ and $a>0$, then by $\left(D_{7}\right)$ the truncation $d \wedge \bar{a}_{X \times X}$ of $d$ by $a$ is in $D^{*}$. By $\left(D_{8}\right)$ we have that $d_{\left(d \wedge \bar{a}_{X \times X}\right)_{x}} \in D$, therefore $\left(d \wedge \bar{a}_{X \times X}\right)_{x} \in \mathcal{M}^{*}(\mathcal{D})$. Note that $\left(d \wedge \bar{a}_{X \times X}\right)_{x} \in \mathbb{F}^{*}(X)$, since $d \wedge \bar{a}_{X \times X} \in D^{*}$. By our hypothesis

$$
0=\left(d \wedge \bar{a}_{X \times X}\right)_{x}(x)=\left(d \wedge \bar{a}_{X \times X}\right)_{x}(y)=\left(d \wedge \bar{a}_{X \times X}\right)(x, y)
$$

therefore $d(x, y)=0$. If $F(D)$ is separating, by Proposition 2.27(i) we get $\forall_{d \in D}(d(x, y)=$ $0) \rightarrow \forall_{h \in \mathcal{M}^{*}(\mathcal{D})}\left(d_{h}(x, y)=0\right) \leftrightarrow \forall_{h \in \mathcal{M}^{*}(\mathcal{D})}(h(x)=h(y)) \rightarrow x=y$.

Proposition 5.3 If $\mathbb{D}=(X, D)$ is a uniform space, then $\rho \mathcal{F}(\mathcal{D})=\mathcal{F}(\rho \mathcal{D})$.

Proof By definition we have that:

$$
\begin{gathered}
\rho \mathcal{F}(\mathcal{D})=\rho\left(X, \mathcal{M}^{*}(\mathcal{D})\right)=\left(\rho X, \rho \mathcal{M}^{*}(\mathcal{D})\right) \\
\mathcal{F}(\rho \mathcal{D})=\mathcal{F}(\rho X, \rho D)=\left(\rho X, \mathcal{M}^{*}(\rho D)\right) \\
\rho \mathcal{M}^{*}(\mathcal{D})=\left\{\rho h \mid h \in \mathcal{M}^{*}(\mathcal{D})\right\}=\left\{\rho h \mid h \in \mathbb{F}^{*}(X), d_{h} \in D\right\} \\
\mathcal{M}^{*}(\rho D)=\left\{h \in \mathbb{F}^{*}(\rho X) \mid d_{h} \in \rho D\right\}
\end{gathered}
$$


Note that by the proof of Proposition 5.2(iii), if $x, y \in X$ then $\forall_{h \in \mathcal{M}^{*}(\mathcal{D})}(h(x)=h(y)) \leftrightarrow$ $\forall_{d \in D}(d(x, y)=0)$, therefore the two equivalence relations $x \approx y \leftrightarrow \forall_{h \in \mathcal{M}^{*}(\mathcal{D})}(h(x)=$ $h(y))$ and $x \sim y \leftrightarrow \forall_{d \in D}(d(x, y)=0)$ are equal, and $\rho X$ is the same set, either if $\rho X$ is the carrier set of $\left(\rho X, \rho \mathcal{M}^{*}(\mathcal{D})\right)$, or of $\left(\rho X, \mathcal{M}^{*}(\rho D)\right)$. First we show that $\rho \mathcal{M}^{*}(\mathcal{D}) \subseteq \mathcal{M}^{*}(\rho D)$. Let $h \in \mathbb{F}^{*}(X)$ such that $d_{h} \in D$. Since $(\rho h)\left([x]_{\sim}\right)=h(x)$, for every $[x]_{\sim} \in \rho X$, we get $\rho h \in \mathbb{F}^{*}(X)$. We need to show that $d_{\rho h} \in \rho D=\{\rho d \mid d \in D\}$. If $[x]_{\sim},[y]_{\sim} \in \rho X$, we have that

$$
\begin{aligned}
d_{\rho h}\left([x]_{\sim},[y]_{\sim}\right) & =\left|(\rho h)\left([x]_{\sim}\right)-(\rho h)\left([y]_{\sim}\right)\right| \\
& =|h(x)-h(y)| \\
& =d_{h}(x, y) \\
& =\left(\rho d_{h}\right)\left([x]_{\sim},[y]_{\sim}\right)
\end{aligned}
$$

ie $d_{\rho h}=\rho d_{h} \in \rho D$, since by hypothesis $d_{h} \in D$. Next we show that $\mathcal{M}^{*}(\rho D) \subseteq$ $\rho \mathcal{M}^{*}(\mathcal{D})$. Let $h^{\prime} \in \mathbb{F}^{*}(\rho X)$ such that $d_{h^{\prime}} \in \rho D$, ie $d_{h^{\prime}}=\rho d$ for some $d \in D$. We define $h: X \rightarrow \mathbb{R}$ by $h(x):=h^{\prime}\left([x]_{\sim}\right)$ for every $x \in X$. Clearly, $h \in \mathbb{F}^{*}(X)$, since $h^{\prime} \in \mathbb{F}^{*}(\rho X)$. If $x, y \in X$, we have that

$$
\begin{aligned}
d_{h}(x, y) & =|h(x)-h(y)| \\
& =\left|h^{\prime}\left([x]_{\sim}\right)-h^{\prime}\left([y]_{\sim}\right)\right| \\
& =d_{h^{\prime}}\left([x]_{\sim},[y]_{\sim}\right) \\
& =(\rho d)\left([x]_{\sim},[y]_{\sim}\right) \\
& =d(x, y)
\end{aligned}
$$

ie $d_{h}=d \in D$, hence $h \in \mathcal{M}^{*}(\mathcal{D})$. Moreover, if $[x]_{\sim} \in \rho X$, we have that $(\rho h)\left([x]_{\sim}\right)=h(x)=h^{\prime}\left([x]_{\sim}\right)$, ie $h^{\prime}=\rho h \in \rho \mathcal{M}^{*}(\mathcal{D})$.

Proposition 5.4 Let $\mathcal{D}=(X, D), \mathcal{E}=(Y, E)$ be uniform spaces.

(i) $\mathcal{M}^{*}(\mathcal{D}) \times \mathcal{M}^{*}(\mathcal{E}) \subseteq \mathcal{M}^{*}(\mathcal{D} \times \mathcal{E})$.

(ii) If $A \subseteq X$ is inhabited, $\mathcal{M}^{*}(D)_{\mid A} \subseteq \mathcal{M}^{*}\left(\mathcal{D}_{\mid A}\right)$.

(iii) If $D_{0} \subseteq \mathbb{D}(X)$ and $M^{*}\left[D_{0}\right]:=\left\{f_{0} \in \mathbb{F}^{*}(X) \mid d_{f_{0}} \in D_{0}\right\}, \bigvee M^{*}\left[D_{0}\right] \subseteq F\left(\coprod D_{0}\right)$.

Proof (i) By definition $\mathcal{M}^{*}(\mathcal{D})=\left\{f^{*} \in \mathbb{F}^{*}(X) \mid d_{f^{*}} \in D\right\}, \mathcal{M}^{*}(\mathcal{E})=\left\{g^{*} \in \mathbb{F}^{*}(Y) \mid\right.$ $\left.d_{g^{*}} \in E\right\}$, and

$$
\mathcal{M}^{*}(\mathcal{D}) \times \mathcal{M}^{*}(\mathcal{E})=\coprod_{d_{f^{*}} \in D}^{d_{g^{*}} \in E} f^{*} \circ \pi_{1}, g^{*} \circ \pi_{2}
$$


Since

$$
\begin{gathered}
\mathcal{M}^{*}(\mathcal{D} \times \mathcal{E})=\left\{h \in \mathbb{F}^{*}(X \times Y) \mid d_{h} \in D \times E\right\}, \\
d_{f^{*}} \in D \rightarrow d_{f^{*}} \odot \pi_{1}=d_{f^{*} \circ \pi_{1}} \in D \times E, \text { and } \\
d_{g^{*}} \in E \rightarrow d_{g^{*}} \odot \pi_{2}=d_{g^{*} \circ \pi_{2}} \in D \times E,
\end{gathered}
$$

we get $\quad\left\{f^{*} \circ \pi_{1} \mid d_{f^{*}} \in D\right\} \cup\left\{g^{*} \circ \pi_{2} \mid d_{g^{*}} \in E\right\} \subseteq \mathcal{M}^{*}(\mathcal{D} \times \mathcal{E})$.

(ii) By definition:

$$
\begin{gathered}
\mathcal{M}^{*}(D)_{\mid A}=\bigvee_{d_{h} \in D}^{h \in \mathbb{F}^{*}(X)} h_{\mid A} \\
\mathcal{M}^{*}\left(\mathcal{D}_{\mid A}\right)=\left\{g \in \mathbb{F}^{*}(A) \mid d_{g} \in D_{\mid A}=\coprod_{d \in D} d_{\mid A \times A}\right\}
\end{gathered}
$$

If $h \in \mathcal{M}^{*}(\mathcal{D})$, then $d_{h} \in D$ and $d_{\left(h_{\mid A}\right)}=\left(d_{h}\right)_{\mid A \times A}$, hence $h_{\mid A} \in \mathcal{M}^{*}\left(\mathcal{D}_{\mid A}\right)$. Consequently, $\left\{h_{\mid A} \mid h \in \mathbb{F}^{*}(X), d_{h} \in D\right\} \subseteq \mathcal{M}^{*}\left(\mathcal{D}_{\mid A}\right)$.

(iii) By Proposition 2.27(i) we have that

$$
f \in F\left(\coprod D_{0}\right)=M^{*}\left(\coprod D_{0}\right) \leftrightarrow d_{f} \in \coprod D_{0} .
$$

If $f_{0} \in M^{*}\left[D_{0}\right]$, then $d_{f_{0}} \in D_{0} \subseteq \coprod D_{0}$, hence $f_{0} \in M^{*}\left(\coprod D_{0}\right)$, ie $M^{*}\left[D_{0}\right] \subseteq$ $M^{*}\left(\coprod D_{0}\right)$, and $\bigvee M^{*}\left[D_{0}\right] \subseteq M^{*}\left(\coprod D_{0}\right)$.

The next proposition has an immediate proof.

Proposition 5.5 Let $\mathcal{D}=(X, D), \mathcal{E}=(Y, E)$ be uniform spaces, and $h \in \mathbb{F}^{*}(X \times Y)$.

(i) If $d \in D$ and $d_{h}=d \odot \pi_{1}$, then $h \in \mathcal{M}^{*}(\mathcal{D}) \times \mathcal{M}^{*}(\mathcal{E})$.

(ii) If $e \in E$ and $d_{h}=e \odot \pi_{2}$, then $h \in \mathcal{M}^{*}(\mathcal{D}) \times \mathcal{M}^{*}(\mathcal{E})$.

Next we relate a Bishop space $\mathcal{F}$ to $\nu(\tau(\mathcal{F}))$ and a uniform space $\mathcal{D}$ to $\tau(\nu(\mathcal{D})$ ).

Proposition 5.6 Let $\mathcal{F}=(X, F)$ be a Bishop space and $\mathcal{D}=(X, D)$ a uniform space.

(i) $F^{*} \subseteq \mathcal{M}^{*}\left(\coprod_{f \in F} d_{f}\right)$.

(ii) $\coprod_{h \in \mathcal{M}^{*}(\mathcal{D})} d_{h} \subseteq D$.

(iii) If $\Phi \subseteq \mathbb{F}^{*}(X)$, then $\coprod_{h \in \mathcal{M}^{*}}\left(\bigsqcup_{f \in \Phi} d_{f}\right) d_{h}=\coprod_{f \in \Phi} d_{f}$. 
Proof (i) If $g \in F^{*}$, then $d_{g} \in \coprod_{f \in F} d_{f}$, and $g \in \mathcal{M}^{*}\left(\coprod_{f \in F} d_{f}\right)$.

(ii) If $h \in \mathcal{M}^{*}(\mathcal{D})$, then $d_{h} \in D$, and the inclusion follows.

(iii) If $g \in \Phi \subseteq \mathbb{F}^{*}(X)$, then $d_{g} \in \coprod_{f \in \Phi} d_{f}$, hence $g \in \mathcal{M}^{*}\left(\coprod_{f \in \Phi} d_{f}\right)$ and $d_{g} \in$ $\coprod_{h \in \mathcal{M}^{*}\left(\amalg_{f \in \Phi} d_{f}\right)} d_{h}$. Consequently,

$$
\coprod_{f \in \Phi} d_{f} \subseteq \coprod_{h \in \mathcal{M}^{*}\left(\coprod_{f \in \Phi} d_{f}\right)} d_{h}
$$

The converse inclusion follows from (ii).

\section{The large uniform space of reals}

Definition 6.1 The pair $\tau(\mathcal{R})=(\mathbb{R}, D(\mathrm{~B}(\mathbb{R})))$, where according to Proposition 4.7

$$
D(\mathrm{~B}(\mathbb{R}))=\coprod_{\phi \in \mathrm{B}(\mathbb{R})} d_{\phi}
$$

is called the large uniform space of reals, and $D(\mathrm{~B}(\mathbb{R}))$ the large uniformity on reals. An $I$-product $\tau(\mathcal{R})^{I}$ of $\tau(\mathcal{R})$ is called a large Euclidean uniform space.

Proposition 6.2 The large uniformity $D(\mathrm{~B}(\mathbb{R}))$ on $\mathbb{R}$ is strictly larger than the metric uniformity $D\left(d_{\mathbb{R}}\right)$ and it is also separating.

Proof Since $^{13} \mathrm{id}_{\mathbb{R}} \in \mathrm{B}(\mathbb{R})$ and $d_{\mathbb{R}}=d_{\left(\mathrm{id}_{\mathbb{R}}\right)}$, we get $D\left(d_{\mathbb{R}}\right) \subseteq D(\mathrm{~B}(\mathbb{R}))$, ie $D(\mathrm{~B}(\mathbb{R}))$ is a larger uniformity on $\mathbb{R}$ than $D\left(d_{\mathbb{R}}\right)$. Since a larger uniformity of a separating one is separating, we conclude that $D(\mathrm{~B}(\mathbb{R}))$ is separating. To show that $D(\mathrm{~B}(\mathbb{R}))$ is strictly larger we use the fact that there are elements of $\mathrm{B}(\mathbb{R})$ which are not in $C_{u}(\mathbb{R})$, the set of real-valued functions that are uniformly continuous on $\mathbb{R}$, like the map $\phi_{0}: \mathbb{R} \rightarrow \mathbb{R}$ defined by $\phi_{0}(x)=\sin \left(x^{2}\right)$ for every $x \in \mathbb{R}$. Hence $d_{\phi_{0}} \in D(\mathrm{~B}(\mathbb{R}))$ but not in $D\left(d_{\mathbb{R}}\right)$, since if $d_{\phi_{0}} \in D\left(d_{\mathbb{R}}\right)$, by Proposition 2.27(i) we get $\phi_{0} \in \mathcal{M}(\mathcal{R})=\operatorname{Mor}\left(\mathcal{D}\left(d_{\mathbb{R}}\right), \mathcal{D}\left(d_{\mathbb{R}}\right)\right.$ ) and by Proposition 2.25(ii) $\phi_{0}$ is uniformly continuous.

Definition 6.3 If $\mathcal{D}=(X, D)$ is a uniform space, we define:

$$
\begin{gathered}
\mathcal{M}_{\tau}(\mathcal{D}):=\operatorname{Mor}(\mathcal{D}, \tau(\mathcal{R}))=\left\{h \in \mathbb{F}(X) \mid \forall_{\phi \in \mathrm{B}(\mathbb{R})}\left(d_{\phi} \odot h=d_{\phi \circ h} \in D\right)\right\} \\
\mathcal{M}_{\tau}^{*}(\mathcal{D}):=\operatorname{Mor}^{*}(\mathcal{D}, \tau(\mathcal{R}))
\end{gathered}
$$

\footnotetext{
${ }^{13}$ It is immediate to see that $\mathrm{B}(\mathbb{R})=\bigvee\left\{\mathrm{id}_{\mathbb{R}}\right\}$.
} 
Proposition 6.4 Let $\mathcal{D}=(X, D)$ be a uniform space.

(i) $\mathcal{M}_{\tau}(\mathcal{D}) \subseteq \mathcal{M}(\mathcal{D})$ and $\mathcal{M}_{\tau}^{*}(\mathcal{D}) \subseteq \mathcal{M}^{*}(\mathcal{D})$.

(ii) $a \in \mathbb{R} \rightarrow \bar{a}_{X} \in \mathcal{M}_{\tau}(\mathcal{D})$.

(iii) If $h \in \mathcal{M}_{\tau}(\mathcal{D}) \rightarrow \phi \in \mathrm{B}(\mathbb{R}) \rightarrow \phi \circ h \in \mathcal{M}_{\tau}(\mathcal{D})$.

Proof (i) If $h \in \mathcal{M}_{\tau}(\mathcal{D})$, then for $\phi=\mathrm{id}_{\mathbb{R}}$ we get $d_{h} \in D$, and by Proposition 2.27(i) $h \in \mathcal{M}(\mathcal{D})$. The inclusion $\mathcal{M}_{\tau}^{*}(\mathcal{D}) \subseteq \mathcal{M}^{*}(\mathcal{D})$ now follows immediately.

(ii) It is immediate from $\phi \circ \bar{a}_{X}=\overline{\phi(a)}_{X}$ and $d_{\overline{\phi(a)} X}=\overline{0}_{X \times X}$.

(iii) If $\theta \in \mathrm{B}(\mathbb{R})$, then $d_{\theta} \odot(\phi \circ h)=d_{\theta \circ(\phi \circ h)}=d_{(\theta \circ \phi) \circ h}=d_{\theta \circ \phi} \odot h \in D$, since $\theta \circ \phi \in \mathrm{B}(\mathbb{R})$ and $h \in \mathcal{M}_{\tau}(\mathcal{D})$.

Proposition 6.5 $\mathcal{M}(\mathcal{R} \times \mathcal{R})$ is strictly larger than $\mathcal{M}_{\tau}(\mathcal{R} \times \mathcal{R})$.

Proof By Proposition 2.28(i) $d_{\mathbb{R}} \in \mathcal{M}(\mathcal{R} \times \mathcal{R})$. We show that $d_{\mathbb{R}}$ does not belong in $\mathcal{M}_{\tau}(\mathcal{R} \times \mathcal{R})$. If that was the case, then

$$
\forall_{\phi \in \mathrm{B}(\mathbb{R})}\left(d_{\phi \circ d_{\mathbb{R}}} \in D\left(d_{\mathbb{R}}\right) \times D\left(d_{\mathbb{R}}\right)\right) .
$$

Let $\phi_{0}(x)=\sin \left(x^{2}\right)$, for every $x \in \mathbb{R}$, for which we know that $d_{\phi_{0}} \in D(\mathrm{~B}(\mathbb{R})) \backslash D\left(d_{\mathbb{R}}\right)$. If $d_{\phi_{0} \circ d_{\mathbb{R}}} \in D\left(d_{\mathbb{R}}\right) \times D\left(d_{\mathbb{R}}\right)$, then, since by Proposition 2.22(i) the mapping $0 i: \mathbb{R} \rightarrow$ $\mathbb{R} \times \mathbb{R}$, defined by $a \mapsto(a, 0)$, for every $a \in \mathbb{R}$, is in $\operatorname{Mor}(\mathcal{R}, \mathcal{R} \times \mathcal{R})$, therefore $d_{\phi_{0} \circ d_{\mathbb{R}}} \odot{ }_{0} i \in D\left(d_{\mathbb{R}}\right)$. If $a, b \in \mathbb{R}$ though, we have that

$$
\begin{aligned}
{\left[d_{\phi_{0} \circ d_{\mathbb{R}}} \odot{ }_{0} i\right](a, b) } & =d_{\phi_{0} \circ d_{\mathbb{R}}}((a, 0),(b, 0)) \\
& =\left|\phi_{0}(|a-0|)-\phi_{0}(|b-0|)\right| \\
& =\left|\sin \left(|a|^{2}\right)-\sin \left(|b|^{2}\right)\right| \\
& =\left|\sin \left(a^{2}\right)-\sin \left(b^{2}\right)\right| \\
& =d_{\phi_{0}}(a, b)
\end{aligned}
$$

ie $d_{\phi_{0} \circ d_{\mathbb{R}}} \odot{ }_{0} i=d_{\phi_{0}} \in D\left(d_{\mathbb{R}}\right)$, which is a contradiction.

Next follows the "large" version of Theorem 3.15.

Theorem 6.6 If $\mathcal{D}=\left(X, \coprod_{f \in \Phi} d_{f}\right)$ is an $\mathrm{f}$-uniform space such that its determining family $\Phi$ is closed under composition with $\mathrm{B}(\mathbb{R})$, then $\mathcal{D}$ is separated if and only if $\mathcal{D}$ is uniformly embedded into the large Euclidean uniform space $\tau(\mathcal{R})^{\Phi}$. 
Proof If $\mathcal{D}$ is separated, the mapping $\varepsilon_{X}: X \rightarrow \mathbb{R}^{\Phi}$, defined in the proof of Theorem 3.15, is an injection. By Proposition 2.21 we get:

$$
\begin{aligned}
D(\mathrm{~B}(\mathbb{R}))^{\Phi}=\left(\coprod_{\phi \in \mathrm{B}(\mathbb{R})} d_{\phi}\right)^{\Phi} & =\coprod_{\phi \in \mathrm{B}(\mathbb{R})}^{f \in \Phi} d_{\phi} \odot \varpi_{f}=\coprod_{\phi \in \mathrm{B}(\mathbb{R})}^{f \in \Phi} d_{\phi \circ \varpi_{f}} \\
\left(D(\mathrm{~B}(\mathbb{R}))^{\Phi}\right)_{\mid \varepsilon_{X}(X)} & =\coprod_{\phi \in \mathrm{B}(\mathbb{R})}\left(d_{\phi \circ \varpi_{f}}\right)_{\mid \varepsilon_{X}(X) \times \varepsilon_{X}(X)}
\end{aligned}
$$

By the $\amalg$-lifting of morphisms we have that:

$$
\varepsilon_{X} \in \operatorname{Mor}\left(\mathcal{D},\left(\tau(\mathcal{R})^{\Phi}\right)_{\mid \varepsilon_{X}(X)}\right) \leftrightarrow \forall_{f \in \Phi} \forall_{\phi \in \mathrm{B}(\mathbb{R})}\left(\left(d_{\phi \circ \varpi_{f}}\right)_{\mid \varepsilon_{X}(X) \times \varepsilon_{X}(X)} \odot \varepsilon_{X} \in \coprod_{f \in \Phi} d_{f}\right)
$$

If $f \in \Phi$ and $\phi \in \mathrm{B}(\mathbb{R})$, then $\phi \circ f \in \Theta$ and

$$
\left(d_{\phi \circ \varpi_{f}}\right)_{\mid \varepsilon_{X}(X) \times \varepsilon_{X}(X)} \odot \varepsilon_{X}=d_{\phi \circ \varpi_{f}} \odot \varepsilon_{X}=d_{\phi \circ f} h
$$

since

$$
\begin{aligned}
{\left[\left(d_{\phi \circ \varpi_{f}}\right)_{\mid \varepsilon_{X}(X) \times \varepsilon_{X}(X)} \odot \varepsilon_{X}\right](x, y) } & =d_{\phi \circ \varpi_{f}}(\hat{x}, \hat{y}) \\
& =\left|\phi\left(\varpi_{f}(\hat{x})\right)-\phi\left(\varpi_{f}(\hat{y})\right)\right| \\
& =|\phi(\hat{x}(f))-\phi(\hat{y}(f))| \\
& =|\phi(f(x))-\phi(f(y))| \\
& =d_{\phi \circ f}(x, y)
\end{aligned}
$$

for every $x, y \in X$. By the above equality we also get

$$
d_{f}=d_{\mathrm{id}_{\mathbb{R}} \circ f}=d_{\mathrm{id}_{\mathbb{R}} \circ \varpi_{f}} \odot \varepsilon_{X}=\left(d_{\mathrm{id}_{\mathbb{R}} \circ \varpi_{f}}\right)_{\mid \varepsilon_{X}(X) \times \varepsilon_{X}(X)} \odot \varepsilon_{X}
$$

for every $f \in \Phi$. By the $\coprod$-lifting of openness $\varepsilon_{X}$ is an open morphism from $\mathcal{D}$ onto $\left(\tau(\mathcal{R})^{\Phi}\right)_{\mid \varepsilon_{X}(X)}$, ie $\varepsilon_{X}$ is a uniform embedding of $\mathcal{D}$ into $\tau(\mathcal{R})^{\Phi}$. The converse follows immediately from Proposition 3.5 and the fact that $D(\mathrm{~B}(\mathbb{R}))$ is separating.

Since a Bishop topology is closed under composition with $B(\mathbb{R})$, we get the following.

Corollary 6.7 If $\mathcal{F}=(X, F)$ is a separated Bishop space, then $\mathcal{D}(\mathcal{F})$ is uniformly embedded into the large Euclidean uniform space $\tau(\mathcal{R})^{F}$.

One can show that the remaining properties of a Bishop topology hold for $\mathcal{M}_{\tau}^{*}(\mathcal{D})$. Although $\mathcal{M}_{\tau}^{*}(\mathcal{D})$ looks smaller than $\mathcal{M}^{*}(\mathcal{D})$, it turns out that the two Bishop topologies are equal, therefore we lose no bounded morphisms of type $X \rightarrow \mathbb{R}$ if we replace the metric uniformity $D\left(d_{\mathbb{R}}\right)$ on $\mathbb{R}$ by the strictly larger uniformity $D(\mathrm{~B}(\mathbb{R}))$. 
Proposition 6.8 If $\mathcal{D}=(X, D)$ is a uniform space then $\mathcal{M}_{\tau}^{*}(\mathcal{D})=\mathcal{M}^{*}(\mathcal{D})$.

Proof It suffices to show $\mathcal{M}^{*}(\mathcal{D}) \subseteq \mathcal{M}_{\tau}^{*}(\mathcal{D})$. By definition, $\mathcal{M}^{*}(\mathcal{D})=\left\{h \in \mathbb{F}^{*}(X) \mid\right.$ $\left.d_{h} \in D\right\}$. Since $\mathrm{B}(\mathbb{R})=\bigvee\left\{\operatorname{id}_{\mathbb{R}}\right\}$ and $d_{h}=d_{\mathbb{R}} \odot h=d_{\operatorname{id}_{\mathbb{R}}} \odot h$, the hypothesis $h \in \mathcal{M}^{*}(\mathcal{D})$ means that $h$ satisfies the required property $d_{\phi} \odot h \in D$ for the subbase $\mathrm{id}_{\mathbb{R}}$ of the topology $\mathrm{B}(\mathbb{R})$. If $a \in \mathbb{R}$, then $d_{\bar{a}_{X}} \odot h=\overline{0}_{X \times X} \odot h=\overline{0}_{X \times X} \in D$. Suppose next that $\phi_{1}, \phi_{2} \in \mathrm{B}(\mathbb{R})$ such that $d_{\phi_{1}} \odot h \in D$ and $d_{\phi_{2}} \odot h \in D$. By Proposition 4.6(ii), property $\left(D_{6}\right)$ and the inductive hypotheses we have that

$$
d_{\phi_{1}+\phi_{2}} \odot h=d_{\left(\phi_{1}+\phi_{2}\right) \circ h}=d_{\left(\phi_{1} \circ h\right)+\left(\phi_{2} \circ h\right)} \leq d_{\phi_{1} \circ h}+d_{\phi_{2} \circ h} \in D
$$

and by $\left(D_{4}\right)$ we get $d_{\phi_{1}+\phi_{2}} \odot h \in D$. If $\theta, \phi \in \mathrm{B}(\mathbb{R})$ and $d_{\phi} \odot h \in D$, then $d_{\theta \circ \phi} \odot h=d_{(\theta \circ \phi) \circ h}=d_{\theta \circ(\phi \circ h)}$. Since $h$ is bounded, $\phi \circ h$ is also bounded; $h(X)$ is bounded, therefore by local compactness of $\left(\mathbb{R}, d_{\mathbb{R}}\right)$ there is a compact subset $K$ of $\mathbb{R}$ such that $h(X) \subseteq K$. Since $\phi$ is uniformly continuous on $K$ we have that $\phi(h(X)) \subseteq \phi(K) \subseteq[-M, M]$ for some $M>0$. By Proposition 4.6(iv) we get

$$
U\left(d_{\phi \circ h}, \omega_{\theta,(\phi \circ h)(X)}(\epsilon), d_{\theta \circ(\phi \circ h)}, \epsilon\right) .
$$

Since $\epsilon>0$ is arbitrary we get $d_{\theta \circ(\phi \circ h)}=d_{\theta} \odot(\phi \circ h) \in D$. Finally, if $\phi, \theta \in \mathrm{B}(\mathbb{R})$ such that $U\left(\phi, \theta, \frac{\epsilon}{3}\right)$ and $d_{\phi} \odot h \in D$, by Proposition 4.6(iii) we get $U\left(d_{\phi}, \frac{\epsilon}{3}, d_{\theta}, \epsilon\right)$, therefore $U\left(d_{\phi} \odot h, \frac{\epsilon}{3}, d_{\theta} \odot h, \epsilon\right)$. Since $\epsilon>0$ is arbitrary, we conclude that $d_{\theta} \odot h \in D$.

Next result shows that the morphism between uniform spaces "captures" Bishop continuity when the large uniform space of reals replaces the uniform space of reals.

Theorem 6.9 $\mathcal{M}(\tau(\mathcal{R}))=\mathrm{B}(\mathbb{R})$ and $\mathcal{M}^{*}(\tau(\mathcal{R}))=\mathrm{B}^{*}(\mathbb{R})$.

Proof By definition we have that

$$
\mathcal{M}(\tau(\mathcal{R}))=\left\{h \in \mathbb{F}(\mathbb{R}) \mid d_{h} \in \coprod_{\phi \in \mathrm{B}(\mathbb{R})} d_{\phi}\right\} .
$$

First we show $\mathrm{B}(\mathbb{R}) \subseteq \mathcal{M}_{\tau}(\tau(\mathcal{R}))$; if $\phi \in \mathrm{B}(\mathbb{R})$, then trivially $d_{\phi} \in \coprod_{\phi \in \mathrm{B}(\mathbb{R})} d_{\phi}$. Next we show that $\mathcal{M}(\tau(\mathcal{R})) \subseteq \mathrm{B}(\mathbb{R})$. We fix some bounded $B \subseteq \mathbb{R}$ and some $\epsilon>0$. Since $d_{h} \in \coprod_{\phi \in \mathrm{B}(\mathbb{R})} d_{\phi}$, there exist $\delta>0, n \in \mathbb{N}$ and $\phi_{1}, \ldots, \phi_{n} \in \mathrm{B}(\mathbb{R})$ such that

$$
\left(d_{\phi_{1}} \vee \ldots \vee d_{\phi_{n}}\right)(x, y) \leq \delta \rightarrow d_{h}(x, y) \leq \epsilon
$$

for every $x, y \in X$. If we define

$$
\omega_{h, B}(\epsilon)=\omega_{\phi_{1}, B}(\delta) \wedge \ldots \wedge \omega_{\phi_{1}, B}(\delta)
$$

then, if $x, y \in B$ such that $|x-y| \leq \omega_{h, B}(\epsilon)$, we get

$$
\left|\phi_{1}(x)-\phi_{1}(y)\right| \leq \delta, \ldots,\left|\phi_{n}(x)-\phi_{n}(y)\right| \leq \delta
$$

therefore $\left(d_{\phi_{1}} \vee \ldots \vee d_{\phi_{n}}\right)(x, y) \leq \delta$. Hence we get $d_{h}(x, y)=|h(x)-h(y)| \leq \epsilon$. The equality $\mathcal{M}^{*}(\tau(\mathcal{R}))=\mathrm{B}^{*}(\mathbb{R})$ follows from the equality $\mathcal{M}(\tau(\mathcal{R}))=\mathrm{B}(\mathbb{R})$. 


\section{Open questions and future work}

In this paper we developed the first steps of a constructive theory of uniformities given by pseudometrics and studied its relation to the constructive theory of Bishop topologies. The interplay between the theory of constructive uniform spaces of pseudometrics and the theory of Bishop topologies is analogous to the interplay between the classical theory of uniform spaces of pseudometrics and the theory of $C(X)$ (see Gillman and Jerison [Chapter 15][21]). The following are some of the many problems and open questions that we want to address in future work.

1. There is more than one way to associate a Bishop topology to a given uniformity of pseudometrics. If $d \in \mathbb{D}(X)$ and $D$ is a uniformity on $X$, we may define the following Bishop topologies on $X$ :

$$
F_{0}(d):=\bigvee_{x \in X} d_{x} \quad F_{0}(D):=\bigvee_{x \in X}^{d \in D} d_{x} \quad F_{0}^{*}(D):=\bigvee_{x \in X}^{d \in D^{*}} d_{x}
$$

Their study is a natural continuation of Section 5 .

2. If $\mathcal{F}=(X, F)$ is a Bishop space, an element $d$ of $\mathbb{D}(X)$ is called $F$-continuous if $d \in \mathcal{M}(\mathcal{F} \times \mathcal{F})$. This notion corresponds to that of a continuous pseudometric on a topological space. We denote by $\mathbb{C D}(F)$ the set of $F$-continuous pseudometrics on $X$. By the $\bigvee$-lifting of Bishop morphisms we get $d \in \mathcal{M}(\mathcal{F} \times \mathcal{F}) \leftrightarrow \operatorname{id}_{\mathbb{R}} \circ d=d \in F \times F$. Moreover, if $f \in F$, then $d_{f} \in \mathbb{C D}(F)$; since $F$ is an algebra and closed under |.| we get

$$
d_{f}=\left|\left(f \circ \pi_{1}\right)-\left(f \circ \pi_{2}\right)\right| \in F \times F .
$$

It would be interesting to study the algebraic and analytic properties of $\mathbb{C D}(F)$.

3. To find a function-theoretic notion of complete uniform space of pseudometrics and to determine those uniform spaces which have a completion.

4. To find a function-theoretic notion of compact uniform space and to connect it to already known notions of compact Bishop spaces found in Petrakis [36, 39].

5. To study constructively extension theorems for pseudometrics, like the classical result that a bounded element of a relative uniformity is extended to a bounded pseudometric in the uniformity of the whole space.

6. To study the uniformities of seminorms and search for appropriate notions of locally convex Bishop spaces.

We would like to thank the anonymous referees for their instructive comments and suggestions. 


\section{References}

[1] S Awodey, Category Theory, Oxford University Press (2010); https://doi.org/10.1093/acprof:oso/9780198568612.001.0001

[2] M J Beeson, Foundations of Constructive Mathematics, Ergebnisse der Mathematik und ihrer Grenzgebiete, Springer Verlag (1985)

[3] J Berger, H Ishihara, E Palmgren, $\mathbf{P}$ Schuster, A predicative completion of a uniform space, Ann. Pure and Appl. Logic 163 (2012) 975-980; https://doi.org/10.1016/j.apal.2011.12.022

[4] E Bishop, Foundations of Constructive Analysis, McGraw-Hill (1967)

[5] E Bishop, Mathematics as a Numerical Language, in [25] (1970) 53-71.

[6] E Bishop, The neat category of stratified spaces, unpublished manuscript (1971)

[7] E Bishop, D S Bridges, Constructive Analysis, Grundlehren der math. Wissenschaften 279, Springer-Verlag, Heidelberg-Berlin-New York (1985); https://doi.org/10.1007/9783-642-61667-9

[8] N Bourbaki, Élements de Mathématique. Topologie générale, Ch. I et II, Hermann \& Cie. Paris (1940)

[9] N Bourbaki, General Topology, Part 2, Ch.5-10 Vol.4 Addison-Wesley (1966)

[10] D S Bridges, F Richman, Varieties of Constructive Mathematics, Cambridge University Press (1987); https://doi.org/10.1017/cbo9780511565663

[11] D S Bridges, S Reeves, Constructive Mathematics in Theory and Programming Practice, Philosophia Mathematica (3) (1999) 65-104; https://doi.org/10.1093/philmat/7.1.65

[12] D S Bridges, L S Vîţă, Techniques of Constructive Analysis, in: Universitext, Springer, New York (2006); https://doi.org/10.1007/978-0-387-38147-3

[13] D S Bridges, L S Vîţă, Apartness and Uniformity: A Constructive Development, in: CiE series "Theory and Applications of Computability", Springer Verlag (2011); https://doi.org/10.1007/978-3-642-22415-7

[14] D S Bridges, Apartness spaces and uniform neighbourhood structures, Annals of Pure and Applied Logic 167 (9) (2016) 850-864; https://doi.org/10.1016/j.apal.2016.04.007

[15] E Bishop, H Cheng, Constructive Measure Theory, Mem. Amer. Math. Soc. 116 (1972)

[16] D S Bridges, Reflections on function spaces, Annals of Pure and Applied Logic 163 (2012) 101-110; https://doi.org/10.1016/j.apal.2011.06.018

[17] G Curi, On the collection of points of a formal space, Ann. Pure Appl. Logic 137 (2006) 126-146; https://doi.org/10.1016/j.apal.2005.05.019

[18] J Dugundji, Topology, Wm. C. Brown Publishers (1989)

[19] C Fox, Point-set and Point-free Topology in Constructive Set Theory, Dissertation, University of Manchester (2005) 
Constructive uniformities of pseudometrics and Bishop topologies

[20] M Fréchet, Sur quelques points du calcul functionnel, Rend. Circ. Mat. di Palermo 22 (1906)

[21] L Gillman, M Jerison, Rings of Continuous Functions, Van Nostrand (1960); https://doi.org/10.1007/978-1-4615-7819-2

[22] N R Howes, Modern Analysis and Topology, Springer-Verlag (1995); https://doi.org/10.1007/978-1-4612-0833-4

[23] H Ishihara, Relating Bishop's function spaces to neighborhood spaces, Annals of Pure and Applied Logic 164 (2013) 482-490; https://doi.org/10.1016/j.apal.2012.10.009

[24] T Kawai, Localic completion of uniform spaces, Logical Methods in Computer Science, September 13 Volume 13 Issue 3 (2017)

[25] A Kino, J Myhill, R E Vesley (Eds.), Intuitionism and Proof Theory, North-Holland (1970); https://doi.org/10.1016/s0049-237x(08)x7036-3

[26] R S Lubarsky, M Rathjen, On the regular extension axiom and its variants, Mathematical Logic Quarterly 49 (5) (2003) 511-518; https://doi.org/10.1002/malq.200310054

[27] S Mac Lane, Categories for the working mathematician, Springer-Verlag (1998); https://doi.org/10.1007/978-1-4757-4721-8

[28] J Myhill, Constructive Set Theory, J. Symbolic Logic 40 (1975) 347-382; https://doi.org/10.2307/2272159

[29] J Pachl, Uniform Spaces and Measures, Fields Institute Monographs 30, Springer Science+Business Media New York (2013); https://doi.org/10.1007/978-1-4614-5058-0

[30] E Palmgren, A constructive and functorial embedding of locally compact metric spaces into locales, Topology Appl., 154 (2007) 1854-1880; https://doi.org/10.1016/j.topol.2007.01.018

[31] I Petrakis, Bishop spaces: constructive point-function topology, in "Mathematisches Forschungsinstitut Oberwolfach Report" No. 52/2014, Mathematical Logic: Proof Theory, Constructive Mathematics (2014) 26-27; https://doi.org/10.4171/OWR/2014/52

[32] I Petrakis, Completely Regular Bishop Spaces, in A. Beckmann, V. Mitrana and M. Soskova (Eds.): Evolving Computability, CiE 2015, LNCS 9136, Springer (2015) 302-312; https://doi.org/10.1007/978-3-319-20028-6_31

[33] I Petrakis, Constructive Topology of Bishop Spaces, PhD Thesis, Ludwig-MaximiliansUniversität, München (2015)

[34] I Petrakis, The Urysohn Extension Theorem for Bishop Spaces, in S. Artemov and A. Nerode (Eds.) Symposium on Logical Foundations of Computer Science 2016, LNCS 9537, Springer (2016) 299-316; https://doi.org/10.1007/978-3-319-27683-0_21

[35] I Petrakis, A direct constructive proof of a Stone-Weierstrass theorem for metric spaces, in A. Beckmann, L. Bienvenu and N. Jonoska (Eds.) Pursuit of the Universal, CiE 2016, LNCS 9709 (2016) 364-374; https://doi.org/10.1007/978-3-319-40189-8_37 
[36] I Petrakis, A constructive function-theoretic approach to topological compactness, Proceedings of the 31st Annual ACM-IEEEE Symposium on Logic in Computer Science (LICS 2016) 605-614; https://doi.org/10.1145/2933575.2933582

[37] I Petrakis, Embeddings of Bishop spaces, submitted (2016)

[38] I Petrakis, A constructive theory of $C^{*}(X)$; part I, in preparation (2019)

[39] I Petrakis, A constructive theory of $C^{*}(X)$; part II, in preparation (2019)

[40] F Richman, Constructive mathematics without choice, in [42], 199-205; https://doi.org/10.1007/978-94-015-9757-9_17

[41] F Richman, Uniform space, unpublished note (2002)

[42] P Schuster, U Berger, H Osswald (eds.), Reuniting the Antipodes Constructive and Nonstandard Views of the Continuum, Proc. 1999 Venice Symposium, Dordrecht: Kluwer (2001); https://doi.org/10.1007/978-94-015-9757-9

[43] E Spanier, Quasi-topologies, Duke Math. J. Volume 30 Number 1 (1963) 1-14; https://doi.org/10.1215/s0012-7094-63-03001-1

[44] B Spitters, Constructive and intuitionistic integration theory and functional analysis, $\mathrm{PhD}$ Thesis, University of Nijmegen (2002)

[45] J W Tukey, Convergence and uniformity in general topology, Annals of Mathematical Studies, Princeton (1940); https://doi.org/10.1515/9781400882199

[46] R C Walker, The Stone-Čech compactification, Springer-Verlag (1974); https://doi.org/10.1007/978-3-642-61935-9

[47] A Weil, Sur les espaces á structure uniforme et sur la topologie générale, Actualities Sci. Ind. 551, Paris (1937)

Mathematisches Institut, Theresienstrasse 39, 80333, Munich, Germany

petrakis@math.lmu.de

http://www . mathematik. uni-muenchen.de/ petrakis/

Received: 16 July 2018 Revised: 5 June 2019 